\title{
Spin Fluctuations in Magnetically Coupled Bi-layer Cuprates
}

\author{
JunIchiro KISHINE* \\ Department of Physics, Faculty of Science, \\ University of Tokyo, 7-3-1 Hongo, Tokyo113. \\ (Received April 10, 1996) \\ Submitted to Journal of the Physical Society of Japan
}

\begin{abstract}
We propose a possible mechanism of pseudo spin gap anomaly(PSGA) in magnetically coupled bi-layer cuprates without any fermion pairing instability. In our proposal PSGA does not necessarily require the spin-charge separation or the breakdown of the Fermi liquid description of a normal state of the cuprate superconductor.

The low energy magnetic excitations are mainly governed by the itinerant nature of the intra-layer system and the inter-layer antiferromagnetic coupling. No matter how weak the bare inter-layer coupling is, it can be dramatically enhanced due to the intra-layer spin fluctuations. As the temperature decreases near the antiferromagnetic phase boundary the strongly enhanced inter-layer correlation induces the inter-layer particle-hole exchange scattering processes that tend to enhance the inter-layer spin singlet formation and kill the triplet formation. We propose that the coupling of spin fluctuations on the adjacend layers via the strong repulsive interaction between parallel spins travelling on each layer give rise to the dynamical screening effects. As a result the low energy part of the spin excitation spectrum is strongly suppressed as the temperature decreases near the antiferromagnetic phase boundary. We ascribe PSGA to this dynamical screening effects.
\end{abstract}

KEYWORDS: magnetically coupled bi-layer, itineracy, spin fluctuations, spin gap

*kishine@watson.phys.s.u-tokyo.ac.jp 


\section{Introduction}

Since the discovery of the high $T_{c}$ cuprate superconductor in 1986, puzzling features in their normal state due to strong correlation [1], especially the spin dynamics of them 2] have provoked a great deal of controversy. In the high $T_{c}$ superconductor, a superconducting phase lies just near the Mott insulating phase where only the spin degree of freedom survives. Thus even in a metallic phase the antiferromagnetic spin fluctuations play essential roles to understand the physical nature of these materials. Especially the spin dynamics of bi-layer cuprates have recently attracted a lot of attention, since the so-called pseudo spin gap anomaly(PSGA), that is one of the most serious problem in this field, seems to be peculiar to bi-layer cuprates.

PSGA was first observed by Yasuoka[3] as the phenomena that NMR longitudinal relaxation rates, $1 / T_{1} T$ deviate from Curie-Weiss like temperature dependence well above the superconducting transition temperature and show a broad peak around the so-called spin gap temperature before it decreases smoothly down through $T_{c}$ with slight change of slope. Up to now the clear signature of PSGA in $1 / T_{1} T$ can be observed in $\mathrm{YBa}_{2} \mathrm{Cu}_{3} \mathrm{O}_{6+x}$ [3, 4, 5, 6, 7, 8, 9, 10, 11] and, $\mathrm{YBa}_{2} \mathrm{Cu}_{4} \mathrm{O}_{8}$ [12, 13, 14], $\mathrm{Y}_{2} \mathrm{Ba}_{4} \mathrm{Cu}_{7} \mathrm{O}_{15}$ 15, $\mathrm{LaBa}_{2} \mathrm{Cu}_{3} \mathrm{O}_{y}$ 16], and $\mathrm{Bi}_{2} \mathrm{Sr}_{2} \mathrm{CaCu}_{2} \mathrm{O}_{y}$ 17, 18 that are all 'bi-layer' cuprates where there are two equivalent $\mathrm{CuO}_{2}$ layer in a unit cell. Furthermore in the lightly doped $\mathrm{YBa}_{2} \mathrm{Cu}_{3} \mathrm{O}_{6+x}$, the inelastic neutron scattering cross sections deviate from a linear $\omega$ dependence in the low energy region and are strongly suppressed above the superconducting transition temperature 19, 20, 21, 22].

On the other hand in typical 'mono-layer' cuprate $\mathrm{La}_{2-x} \mathrm{Sr}_{x} \mathrm{CuO}_{4}, 1 / T_{1} T$ begin to deviate slightly from Curie-Weiss law below $T \sim 200 \mathrm{~K} 23$, but do not show clear peak structure above $T_{c}$. As for the neutron experiments on LSCO system, only recently an overall energy spectrum of magnetic fluctuations was reported 24] in homogeneous single crystal of $\mathrm{La}_{1.85} \mathrm{Sr}_{0.15} \mathrm{CuO}_{4}$. They first reported the gap formation in LSCO system below $T_{c}$ and succeeded in detecting the superconducting phase of this system. However they didn't detect the pseudo-spin gap anomaly in this compound. Furthermore the single layer over-doped cuprate $\mathrm{Tl}_{2} \mathrm{Ba}_{2} \mathrm{CuO}_{y}$ exhibits a very broad peak in both of $1 / T_{1} T$ and $1 / T_{2}$ slightly above $T_{c}$. As Yasuoka pointed out 16, 25, 26, this behavior should be atributed to the band effects and cannnot be regarded as the spin-gap behavior. These experimental facts indicate that PSGA is peculiar to bilayer cuprates.

The most important feature of PSGA is that the strong suppression of the low energy spin excitations does not accompany the clear gap like structure in the low energy charge excitations. By reason of this fact, the spin gap in high $T_{c}$ superconductor is called the pseudo gap. The suppression of the spin excitations clearly indicates that the singlet pair formation is favored and as a result the magnetic degree of freedom tends to be killed [27]. Therefore to understand PSGA phenomena we have to seek some mechanism that favors the singlet pair formation without any suppression of the charge excitations.

From theoretical viewpoints there are two fixed points to describe low energy ex- 
citations in a normal state of the cuprate superconductor. One fixed point is the Fermi liquid fixed point where the low energy excitations are described in terms of quasi-paricle excitations. If we try to explain PSGA within this framework, we have to seek a mechanism that enhances the singlet pair formation without any pairing instability since pairing instability in a quasi-particle system means the superconducting transition where both of the spin and charge degree of freedom condensate into pair. Another fixed point is the non-Fermi liquid picture with spin charge separation where the low energy excitations are spin 1/2 chargeless fermions(spinons) and charged spinless bosons(holons) [1]. In this picture PSGA is ascribed to the condensation of the spinons into the singlet pair.

The Hubbard model is the simplest model to describe the effects of the strong correlation within the Fermi liquid picture. In the Hubbard model, we take into account the electronic correlations by including the on-site repulsion, $U$, between two electrons with opposite spins. Then the simplest scheme to describe the collective spin excitations is the random phase approximation(RPA), namely paramagnon theory[28]. Apart from PSGA, quantitative behavior of dynamical spin fluctuations can well be described within the simple RPA [29, 30, 31]. We can go beyond RPA by treating the feedback effects of the spin fluctuations on the irreducible polarization in a self consistent manner 32, 33, 34. More sophisticated treatment based on Baym-Kadanoff formalism that treats the exchange of fluctuations in the particle-hole and particle-particle channels in a self consistent manner has been proposed 35, 36]. Phenomenological approach to the antiferromagnetic spin fluctuations proposed by Millis, Monien and Pines 37 also lays its microscopic foundation on the Fermi liquid picture. However it seems difficult to explain PSGA based only on the Hubbard model, since the Hubbard interaction never distinguishes the singlet and triplet pair selectively. In the Hubbard model there appear only one scattering channel between quasi-particles with opposite spins and as a result it cannot describe the tendency of the enhanced singlet.

On the other hand the non-Fermi liquid picture with spin-charge separation stems from the $t-J$ model that can be regarded as the strong coupling limit of the Hubbard model 38. This model can give magnetic phase diagram within the mean field level [39]. Recently the extention of the $t-J$ model to the bi-layer cuprates was also reported 40, 41, 46]. In the $t-J$ model the presence of the spin dependent interaction is essential. It produces three fundamental scattering channels, one with spin-flip and two without spin-flip. These scattering channels can be decomposed into the singlet and triplet channel and spin dependent nature of scattering process can well be described. Concerning PSGA there have been some proposals mainly from the gauge field theoretical approach based on the $t-J$ model [42, 43, 44, 45, 46, 47]. Altshuler and Ioffe 45 first pointed out the importance of bi-layer correlation. They claimed that if the spins on each layer form a spin liquid state, this transition can be described as a spinon pairing on adjacent layers, that leads to the spin-gap anomaly. As for the spinon condensation into the singlet pair, Ubbens and Lee claimed [44] that $U(1)$ gauge fluctuations tend to destroy the intra-layer spinon pairing, although 
it is less effective in destroying the inter-layer pairing [46. However recently Wen and Lee [47 reported that the spin gap phase is best understood as the intra-layer staggered flux phase where the $S U(2)$ symmetry is preserved away from half filling. Therefore within the framework of 2-dimensional gauge theory there still exist some ambiguity on whether PSGA is intrinsic to bi-layer structure or not.

However it remains an unsettled question whether the low energy excitations in cuprate can be described as the Fermi liquid or not. In the present paper, we should like to explore a different possibility to understand PSGA without any fermion condensation, which has never been examined. As stated above to understand PSGA the existence of spin dependent interaction is essentially important. However this does not necessary mean the spin-charge separation really takes place. Motivated by this consideration, we try to understand PSGA without breakdown of the Fermi liquid description. To compensate the drawback of the Hubbard model that it cannot distinguish the singlet pair formation selectively, we consider the magnetically coupled bi-layer cuprates and try to understand the PSGA without any pairing instability. Our motivation was first provoked by the idea by Ioffe et.al 18 , where the strongly developed inter-layer spin fluctuations dramatically enhance the inter-layer interaction. In the present paper we treat the intra-layer spin fluctuations based on the Hubbard model. As the temperature decreases, the developed intra-layer spin fluctuations dramatically enhance the inter-layer magnetic correlation near the magnetic phase boundary. Therefore no matter how weak the bare inter-layer magnetic coupling is, it can affect the whole structure of the magnetic excitations of the system at the low temperature near the magnetic phase boundary.

Just near the antiferromagnetic phase boundary, the enhanced inter-layer correlations strongly induce the inter-layer particle-hole exchange scattering processes. In this paper we show that due to these scattering processes the inter-layer singlet formation is strongly enhanced while the triplet formation is strongly suppressed. In other words interaction between parallel spins becomes strongly repulsive. As a result we can ascribe the PSGA to the dynamical screening of the low energy spin excitations due to this repulsion. Actually we can show that as the temperature decreases and the intra-layer spin fluctuations develop, the imaginary parts of the whole susceptibility reveal apparent gap-like structure in the low energy region. We can assign the PSGA to this mechanism.

The outline of this paper is as follows. In section 2, we present the model and formalism. In section 3, we present how dramatically the inter-layer bare interaction is enhanced by the intra-layer spin fluctuations. We pay our attention to the spin rotational invariance relation. In section 4 , we investigate the inter-layer particle-hole exchange scattering processes driven by the enhanced inter-layer intetaction in some detail. In section 5, we show that the coupling of spin fluctuations on the adjacend layers via the inter-layer processes give rise to the dynamical screening effects. In section 6, we present the relations between microscopic spin correlations obtained in the present approach and experimentally observed quantities in magnetically coupled bi-layer cuprates. In section 7 , We present the numerical results on the analytically 
continued dynamical susceptibilities and show that our proposal can actually be realized.

\section{Model and Formalism}

In this section we present our model and basic formalism to treat the spin fluctuations in magnetically coupled bi-layer. We consider $\mathrm{YBa}_{2} \mathrm{Cu}_{3} \mathrm{O}_{6+x}$ (Y123) compounds as representatives of the bi-layer family. As shown in Fig. 1. there are a pair of equivalent $\mathrm{CuO}_{2}$ layers per unit cell that are separated by the charge reservoir layers. We ignore a small orthorhombic distortion of the $\mathrm{CuO}_{2}$ lattice. The distance between the adjacent $\mathrm{CuO}_{2}$ layers is approximately $c^{*} \sim 3.2 \boldsymbol{r} \mathrm{A}$. On the other hand the distance between the pair of layers is approximately $c \sim 8.2 \boldsymbol{r} \mathrm{A}$.

\subsection{Model Hamiltonian}

We start with the Hubbard Hamiltonian with small direct inter-layer hopping

$$
\mathcal{H}=\sum_{i, j} \sum_{\sigma} \sum_{n} t_{i j} c_{n, j, \sigma}^{\dagger} c_{n, i, \sigma}+t_{\perp} \sum_{\sigma} \sum_{n \neq m} c_{n, i, \sigma}^{\dagger} c_{m, i, \sigma}+U \sum_{i} \sum_{n} n_{n, i, \uparrow} n_{n, i, \downarrow}
$$

where $c_{n, i, \sigma}^{\dagger}\left(c_{n, i, \sigma}\right)$ is a creation (annihilation) operator of an itinerant carrier belonging to the $i$-th $\mathrm{Cu}$ site of the $n$-th layer $(n=1,2)$ with the spin projection $\sigma$ and $n_{n, i, \sigma}=c_{n, i, \sigma}^{\dagger} c_{n, i, \sigma}$. Furthermore $t_{i j}$ denotes the intra-layer hopping integral between the $i$ - $j$ sites and $t_{\perp}$ denotes the inter-layer hopping integral. As for the intra-layer on-site Hubbard repulsion, $U$, we assume the intermediate coupling scheme, namely $U \sim 3 t$, where $t$ is the hopping integral between the nearest neighbor sites. On the other hand, we can safely assume, $t_{\perp} \ll U$ and therefore we can treat the direct inter-layer hopping process in a $t$ - $J$ like manner. Thus we obtain

$$
\mathcal{H}=\mathcal{H}_{/ /}+\mathcal{H}_{\perp}
$$

where

$$
\mathcal{H}_{/ /}=\sum_{i, j} \sum_{\sigma} \sum_{n} t_{i j} c_{n, j, \sigma}^{\dagger} c_{n, i, \sigma}+U \sum_{i} \sum_{n} n_{n, i, \uparrow} n_{n, i, \downarrow}
$$

denotes the intra-layer Hubbard Hamiltonian and

$$
\mathcal{H}_{\perp}=t_{\perp} \sum_{\sigma} \sum_{n \neq m}\left(1-n_{n, i,-\sigma}\right) c_{n, i, \sigma}^{\dagger} c_{m, i, \sigma}\left(1-n_{m, i,-\sigma}\right)+2 J_{0 \perp} \sum_{i} \sum_{m \neq n} \boldsymbol{S}_{m, i} \cdot \boldsymbol{S}_{n, i},
$$

is the $t-J$ like counterpart that gives rise to the inter-layer magnetic coupling. The spin fluctuation operator is introduced by

$$
\boldsymbol{S}_{i}^{(n)}=\frac{1}{2} \sum_{\alpha \beta} c_{n, i, \alpha}^{\dagger} \boldsymbol{\sigma}_{\alpha \beta} c_{n, i, \beta},
$$

where $\boldsymbol{\sigma}$ denotes usual Pauli matrices and we set $\hbar=1$. We note that the hopping term in $\mathcal{H}_{\perp}$ depends on the carrier density and near the antiferromagnetic phase 
boundary it is reduced, while the magnetic coupling is unlikely to be sensitive to the doping. Furthermore, as will be shown later, near the phase boundary the inter-layer coupling is dramatically enhanced by the intra-layer spin fluctuations. Based on this fact, in $\mathcal{H}_{\perp}$ we keep only the magnetic term.

Thus we are lead to the assumption that the spin fluctuations in bi-layer cuprates can well be described by the effective Hamiltonian

$$
\mathcal{H}_{/ /}=\sum_{i, j} \sum_{\sigma} \sum_{n} t_{i j} c_{n, j, \sigma}^{\dagger} c_{n, i, \sigma}+U \sum_{i} \sum_{n} n_{n, i, \uparrow} n_{n, i, \downarrow},
$$

and

$$
\mathcal{H}_{\perp}^{e f f}=2 J_{0 \perp} \sum_{i} \sum_{m \neq n} \boldsymbol{S}_{m, i} \cdot \boldsymbol{S}_{n, i} .
$$

By taking the lattice Fourier transform

$$
c_{n, i, \sigma}=\frac{1}{\sqrt{N}} \sum_{\boldsymbol{k}} \mathrm{e}^{i \boldsymbol{k} \cdot \mathbf{i}} c_{n, \boldsymbol{k}, \sigma},
$$

the effective Hamiltonian can be written by

$$
\begin{array}{r}
\mathcal{H}_{/ /}=\sum_{\boldsymbol{k}} \sum_{\sigma} \sum_{n} \varepsilon_{n, \boldsymbol{k}_{n,}} c_{n, \sigma}^{\dagger} \boldsymbol{k}_{n, \boldsymbol{k}, \sigma}^{c_{n}} \\
+\frac{U}{N} \sum_{\boldsymbol{k}, \boldsymbol{k}^{\prime}, \boldsymbol{q}} \sum_{n=1,2} c_{n, \boldsymbol{k}+\boldsymbol{q}, \uparrow}^{\dagger} c_{n, \boldsymbol{k}, \uparrow} c_{n, \boldsymbol{k}^{\prime}-\boldsymbol{q}, \downarrow}^{c_{n}, \boldsymbol{k}^{\prime}, \downarrow}
\end{array}
$$

and

$$
\mathcal{H}_{\perp}^{e f f}=2 J_{0 \perp} \sum_{\boldsymbol{q}} \sum_{m \neq n} \boldsymbol{S}_{m}(\boldsymbol{q}) \cdot \boldsymbol{S}_{n}(-\boldsymbol{q}) .
$$

where $N$ is the number of the lattice sites and

$$
\boldsymbol{S}_{n}(\boldsymbol{q})=\frac{1}{2} \sum_{\boldsymbol{k}} \sum_{\alpha \beta} c_{n, \boldsymbol{k}+\boldsymbol{q}, \alpha}^{\dagger} \boldsymbol{\sigma}_{\alpha \beta} c_{n, \boldsymbol{k}, \beta},
$$

denotes the spin fluctuation in the momentum space. Here we note the momentum $\boldsymbol{k}$ and $\boldsymbol{q}$ are the two-dimensional momentum since in our model the itinerant carriers are confined into a single plane. In the kinetic term, we have included the hopping process to the second nearest neighbor sites

$$
\begin{array}{r}
\varepsilon_{1, \boldsymbol{k}}=\varepsilon_{2, \boldsymbol{k}} \equiv \varepsilon_{\boldsymbol{k}}=-2 t\left(\cos k_{x} a+\cos k_{y} a\right)-2 t^{\prime} \cos k_{x} a \cos k_{y} a \\
-2 t^{\prime \prime}\left(\cos 2 k_{x} a+\cos 2 k_{y} a\right),
\end{array}
$$

where we put $t^{\prime}=-t / 5$ and $t^{\prime \prime}=t / 4$ to reproduce the Fermi contour of YBCO system[41, 49].

Now we briefly comment on the experimental evidences for the inter-layer magnetic coupling. It is well established that there remains the strong intra-layer antiferromagnetic spin-spin coupling which is estimated as $J_{0 / /}=80_{-30}^{+60} \mathrm{meV}$ from the 
neutron experiments [50]. On the other hand, although existence of the inter-layer antiferromagnetic spin-spin interaction has been widely accepted, question about the magnitude of this interaction had been under debates. Concerning this issue, the early neutron scattering experiment gave very weak strength of inter-layer magnetic coupling $J_{0 \perp} \sim 0.06 J_{0 / /}$. This estimation was based on the fact that the expected optical gap of spin wave excitation, $2 \sqrt{J_{0 / /} J_{0 \perp}}$, in an insulating phase had never been observed even up to $60 \mathrm{meV}[51]$. However recently it was reproted that midinfrared transmission and reflection mesurements picked up the optical branch at $178.0 \mathrm{meV}[52]$ and therefore $J_{0 \perp}$ can be estimated as $J_{0 \perp} \sim 0.55 J_{0 / /}$. Moreover recent NMR cross-relaxation mesurements of $\mathrm{Y}_{2} \mathrm{Ba}_{4} \mathrm{Cu}_{7} \mathrm{O}_{15}$ [15] also suggest that the inter-layer spin-spin coupling can reach $J_{0 \perp} \sim 0.25 J_{0 / /}$. Tus we can say that the importance of the inter-layer magnetic coupling should be duely recognized to understand the magnetic properties of bi-layer cuprates.

\subsection{Dynamical Spin Susceptibility}

In the case of magnetically coupled bi-layer, the dynamical spin susceptibility can be written in the thermal Green's function formalism as

$$
\begin{aligned}
\chi_{m n}^{\alpha \beta}\left(\boldsymbol{q} ; i \omega_{l}\right) & =\int_{0}^{1 / T} d \tau e^{i \omega_{l} \tau}<T_{\tau}\left[S_{m}^{\alpha}(\boldsymbol{q}, \tau) S_{n}^{\beta}(-\boldsymbol{q}, 0)\right]> \\
& =\frac{1}{4} \sum_{\mu \nu \lambda \rho} \sigma_{\mu \nu}^{\alpha} \Gamma_{m n}^{\mu \nu ; \lambda \rho}\left(\boldsymbol{q} ; i \omega_{l}\right) \sigma_{\lambda \rho}^{\beta},
\end{aligned}
$$

where

$$
\Gamma_{m n}^{\mu \nu ; \lambda \rho}\left(\boldsymbol{q} ; i \omega_{l}\right)=\int_{0}^{1 / T} d \tau e^{i \omega_{l} \tau} \frac{1}{N} \sum_{\boldsymbol{k}, \boldsymbol{k}^{\prime}}<T_{\tau}\left[c_{m, \boldsymbol{k}^{\prime}, \mu}^{\dagger}(\tau) c_{m, \boldsymbol{k}^{\prime}+\boldsymbol{q}, \nu}(\tau) c_{n, \boldsymbol{k}+\boldsymbol{q}, \rho}^{\dagger}(0) c_{n, \boldsymbol{k}, \lambda}(0)\right]>
$$

denotes the spin dependent polarization function. Now $m, n$ are layer indices, $\alpha, \beta=$ ,,$+- z$ are the spin indices, $\mu, \nu, \lambda, \rho=\uparrow, \downarrow$ are the spin projection, and $\omega_{l}=2 \pi T l$ is a bosonic Matsubara frequency. Furthermore

$$
c_{n, \boldsymbol{k}, \alpha}^{\dagger}(\tau)=\exp (\tau \mathcal{H}) c_{n, \boldsymbol{k}, \alpha}^{\dagger}(0) \exp (-\tau \mathcal{H})
$$

represent imaginary time dependent creation operator where we set $\hbar=1$ and $k_{B}=$ 1. The thermal average $\langle\cdots\rangle \equiv \operatorname{Tr}\left(\mathrm{e}^{-\mathcal{H} / T} \cdots\right) / \operatorname{Tre}^{-\mathcal{H} / T}$ is taken under the full Hamiltonian. Due to the symmetry under exchange of layers, the spin susceptibility $\chi_{m n}^{\alpha \beta}$ has only two independent components with respect to $m, n$ : the intra-layer (diagonal) correlation $\chi_{11}^{\alpha \beta}=\chi_{22}^{\alpha \beta} \equiv \chi_{/ /}^{\alpha \beta}$ and the inter-layer (off-diagonal) correlation $\chi_{12}^{\alpha \beta}=\chi_{21}^{\alpha \beta^{*}} \equiv \chi_{\perp}^{\alpha \beta}$. Furthermore each element has two independent components in spin space: transverse counterpart $\chi_{m n}^{+-}$and longitudinal counterpart $\chi_{m n}^{z z}$, which are given by

$$
\left\{\begin{array}{c}
\chi_{m n}^{+-}\left(\boldsymbol{q} ; i \omega_{l}\right)=\Gamma_{m n}^{\uparrow \downarrow ; \downarrow \uparrow}\left(\boldsymbol{q} ; i \omega_{l}\right), \\
\chi_{m n}^{z z}\left(\boldsymbol{q} ; i \omega_{l}\right)=\frac{1}{4}\left[\Gamma_{m n}^{\uparrow \uparrow ; \uparrow \uparrow}\left(\boldsymbol{q} ; i \omega_{l}\right)+\Gamma_{m n}^{\downarrow \downarrow ; \downarrow \downarrow}\left(\boldsymbol{q} ; i \omega_{l}\right)-\Gamma_{m n}^{\uparrow \uparrow ; \downarrow \downarrow}\left(\boldsymbol{q} ; i \omega_{l}\right)-\Gamma_{m n}^{\downarrow \downarrow ; \uparrow \uparrow}\left(\boldsymbol{q} ; i \omega_{l}\right)\right] .
\end{array}\right.
$$


Throughout this paper we consider the paramagnetic pahse and therefore the dynamical spin susceptibilities must satisfy the rotational symmetry relation in spin space;

$$
2 \chi_{m n}^{z z}\left(\boldsymbol{q} ; i \omega_{l}\right)=\chi_{m n}^{+-}\left(\boldsymbol{q} ; i \omega_{l}\right) .
$$

We should always check this relation when we carry the theoretical investigation a stage further. Since we assumed there is no inter-layer carrier hopping, the noninteracting counterpart can simply be written as

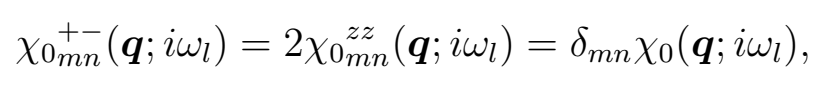

where

$$
\begin{array}{r}
\chi_{0}\left(\boldsymbol{q} ; i \omega_{l}\right)=-\frac{T}{N} \sum_{i \varepsilon_{n}} \sum_{\boldsymbol{k}} \mathcal{G}_{0}\left(\boldsymbol{k}+\boldsymbol{q} ; i \varepsilon_{n}+\omega_{l}\right) \mathcal{G}_{0}\left(\boldsymbol{k} ; i \varepsilon_{n}\right) \\
=\frac{1}{2 N} \sum_{\boldsymbol{k}} \frac{\Lambda_{\boldsymbol{k}, \boldsymbol{q}}(T)\left(\xi_{\boldsymbol{k}+\boldsymbol{q}}-\xi_{\boldsymbol{k}}\right)}{\omega_{l}^{2}+\left(\xi_{\boldsymbol{k}+\boldsymbol{q}}-\xi_{\boldsymbol{k}}\right)^{2}} .
\end{array}
$$

Now

$$
\mathcal{G}_{0}\left(\boldsymbol{k} ; i \varepsilon_{n}\right)=\frac{1}{i \varepsilon_{n}-\xi_{\boldsymbol{k}}}
$$

is a Green's function for a free carrier where $\varepsilon_{n}=(2 n+1) \pi T$ is a fermionic Matsubara frequency. The thermal extinction factor is given by

$$
\Lambda_{\boldsymbol{k}, \boldsymbol{q}}(T)=\tanh \left(\frac{\xi_{\boldsymbol{k}+\boldsymbol{q}}}{2 T}\right)-\tanh \left(\frac{\xi_{\boldsymbol{k}}}{2 T}\right),
$$

where $\xi_{\boldsymbol{k}}=\varepsilon_{\boldsymbol{k}}-\mu$.

First we consider the intra-layer spin fluctuations driven by the Hubbard interaction within the frame work of random phase approximation (RPA). Taking into the effects of the interaction, instead of (20), the dynamical susceptibility is rewritten in a form,

$$
\chi^{\alpha \beta}\left(\boldsymbol{q} ; i \omega_{l}\right)=-\frac{T}{N} \sum_{k} \mathcal{G}_{0}\left(\boldsymbol{k}+\boldsymbol{q} ; i \varepsilon_{n}+\omega_{l}\right) \gamma^{\alpha \beta}\left(\boldsymbol{q} ; i \omega_{l}\right) \mathcal{G}_{0}\left(\boldsymbol{k} ; i \varepsilon_{n}\right),
$$

where $\gamma^{\alpha \beta}(q)$ denotes the RPA triangle vertex. Usual RPA for Hubbard model[28], gives

$$
\left\{\begin{array}{c}
\gamma^{+-}\left(\boldsymbol{q} ; i \omega_{l}\right)=\frac{1}{1-U \chi_{0}\left(\boldsymbol{q} ; i \omega_{l}\right)}, \\
\gamma^{\sigma \sigma}\left(\boldsymbol{q} ; i \omega_{l}\right)=\frac{1}{1-U^{2} \chi_{0}\left(\boldsymbol{q} ; i \omega_{l}\right)^{2}}, \\
\gamma^{\sigma,-\sigma}\left(\boldsymbol{q} ; i \omega_{l}\right)=-\frac{U \chi_{0}\left(\boldsymbol{q} ; i \omega_{l}\right)}{1-U^{2} \chi_{0}\left(\boldsymbol{q} ; i \omega_{l}\right)^{2}} .
\end{array}\right.
$$


These vertices satisfy the spin-rotational invariance relation

$$
\gamma^{\sigma \sigma}\left(\boldsymbol{q} ; i \omega_{l}\right)-\gamma^{\sigma,-\sigma}\left(\boldsymbol{q} ; i \omega_{l}\right)=\gamma^{+-}\left(\boldsymbol{q} ; i \omega_{l}\right) \equiv \gamma\left(\boldsymbol{q} ; i \omega_{l}\right),
$$

where $\gamma\left(\boldsymbol{q} ; i \omega_{l}\right) \equiv\left[1-U \chi_{0}\left(\boldsymbol{q} ; i \omega_{l}\right)\right]^{-1}$ is a Stoner factor. These vertices give the dynamical spin susceptibilities;

$$
\left\{\begin{array}{c}
\chi^{+-}\left(\boldsymbol{q} ; i \omega_{l}\right)=\chi_{0}\left(\boldsymbol{q} ; i \omega_{l}\right) \gamma^{+-}\left(\boldsymbol{q} ; i \omega_{l}\right) \\
\chi^{\sigma \sigma}\left(\boldsymbol{q} ; i \omega_{l}\right)=\chi_{0}\left(\boldsymbol{q} ; i \omega_{l}\right) \gamma^{\sigma \sigma}\left(\boldsymbol{q} ; i \omega_{l}\right) \\
\chi^{\sigma,-\sigma}\left(\boldsymbol{q} ; i \omega_{l}\right)=\chi_{0}\left(\boldsymbol{q} ; i \omega_{l}\right) \gamma^{\sigma,-\sigma}\left(\boldsymbol{q} ; i \omega_{l}\right) \\
\chi^{z z}\left(\boldsymbol{q} ; i \omega_{l}\right)=\frac{1}{4} \sum_{\sigma}\left[\chi^{\sigma, \sigma}\left(\boldsymbol{q} ; i \omega_{l}\right)-\chi^{\sigma,-\sigma}\left(\boldsymbol{q} ; i \omega_{l}\right)\right]
\end{array}\right.
$$

where we used the relation (16). We note that in this step the spin rotational symmetry relation

$$
\chi^{+-}\left(\boldsymbol{q} ; i \omega_{l}\right)=2 \chi^{z z}\left(\boldsymbol{q} ; i \omega_{l}\right) \equiv \chi\left(\boldsymbol{q} ; i \omega_{l}\right),
$$

is satisfied where we define the RPA spin susceptibility

$$
\chi\left(\boldsymbol{q} ; i \omega_{l}\right)=\frac{\chi_{0}\left(\boldsymbol{q} ; i \omega_{l}\right)}{1-U \chi_{0}\left(\boldsymbol{q} ; i \omega_{l}\right)} .
$$

\section{Enhanced Inter-layer Interaction}

The fundamental processes induced by the inter-layer interaction are given through the decomposition

$$
\boldsymbol{S}_{1} \cdot \boldsymbol{S}_{2}=\frac{1}{2}\left(S_{1}^{+} S_{2}^{-}+S_{1}^{-} S_{2}^{+}\right)+S_{1}^{z} S_{2}^{z}
$$

By rewriting this expression in a second quantized form, we get three scattering processes between propagating spins on each layer. The first term of (28) produces the scattering process with spin flip (Type-A). The second term of (28) produces two fundamental processes; scattering processes between parallel spins (Type-B) and between anti-parallel spins (Type-C). These processes are shown in Fig. 2. These three scattering processes can couple to the intra-layer spin fluctuations in each of adjacent layers. In Fig. 3. we show how the inter-layer exchange interaction vertices couple to the intra-layer RPA spin fluctuations. Thus the intra-layer spin fluctuations on each layer can couple to each other via these scattering vertices as shown in Fig. 4. As is shown in Fig. 4 (a), the Type-A scattering can couple only to the intra-layer transverse spin fluctuations. Further Type-B and Type-C scattering can couple only to the intra-layer longitudinal spin fluctuations, as depicted in Fig. 4(b),(c). Then the enhanced inter-layer interaction for each channel can be given respectively by:

$$
\frac{1}{2} J_{\perp}^{+-}\left(\boldsymbol{q} ; i \omega_{l}\right)=\gamma^{+-}\left(\boldsymbol{q} ; i \omega_{l}\right) \frac{J_{0 \perp}}{2} \gamma^{+-}\left(\boldsymbol{q} ; i \omega_{l}\right)=\frac{J_{0 \perp}}{2} \gamma\left(\boldsymbol{q} ; i \omega_{l}\right)^{2}
$$


for the type-A scattering and

$$
\begin{aligned}
\frac{1}{4} J_{\perp}^{\sigma \sigma}\left(\boldsymbol{q} ; i \omega_{l}\right)= & \gamma^{\sigma,-\sigma}\left(\boldsymbol{q} ; i \omega_{l}\right) \frac{J_{0 \perp}}{4} \gamma^{-\sigma, \sigma}\left(\boldsymbol{q} ; i \omega_{l}\right)-\gamma^{\sigma, \sigma}\left(\boldsymbol{q} ; i \omega_{l}\right) \frac{J_{0 \perp}}{4} \gamma^{\sigma, \sigma}\left(\boldsymbol{q} ; i \omega_{l}\right) \\
& -\gamma^{\sigma, \sigma}\left(\boldsymbol{q} ; i \omega_{l}\right) \frac{J_{0 \perp}}{4} \gamma^{-\sigma, \sigma}\left(\boldsymbol{q} ; i \omega_{l}\right)+\gamma^{\sigma, \sigma}\left(\boldsymbol{q} ; i \omega_{l}\right) \frac{J_{0 \perp}}{4} \gamma^{\sigma, \sigma}\left(\boldsymbol{q} ; i \omega_{l}\right) \\
= & \frac{J_{0 \perp}}{4} \gamma\left(\boldsymbol{q} ; i \omega_{l}\right)^{2},
\end{aligned}
$$

and for the type-B scattering

$$
\begin{aligned}
-\frac{1}{4} J_{\perp}^{\sigma,-\sigma}\left(\boldsymbol{q} ; i \omega_{l}\right) & =-\gamma^{\sigma,-\sigma}\left(\boldsymbol{q} ; i \omega_{l}\right) \frac{J_{0 \perp}}{4} \gamma^{\sigma,-\sigma}\left(\boldsymbol{q} ; i \omega_{l}\right)+\gamma^{\sigma,-\sigma}\left(\boldsymbol{q} ; i \omega_{l}\right) \frac{J_{0 \perp}}{4} \gamma^{-\sigma,-\sigma}\left(\boldsymbol{q} ; i \omega_{l}\right) \\
& +\gamma^{\sigma, \sigma}\left(\boldsymbol{q} ; i \omega_{l}\right) \frac{J_{0 \perp}}{4} \gamma^{\sigma,-\sigma}\left(\boldsymbol{q} ; i \omega_{l}\right)-\gamma^{\sigma, \sigma}\left(\boldsymbol{q} ; i \omega_{l}\right) \frac{J_{0 \perp}}{4} \gamma^{-\sigma,-\sigma}\left(\boldsymbol{q} ; i \omega_{l}\right) \\
& =-\frac{J_{0 \perp}}{4} \gamma\left(\boldsymbol{q} ; i \omega_{l}\right)^{2},
\end{aligned}
$$

for the type-C scattering. Thus scattering vertices corresponding to each channel are enhanced in the same manner. This situation comes from the rotational symmetry of the interaction in the spin space. Thus we introduce the enhanced inter-layer interaction

$$
J_{\perp}\left(\boldsymbol{q} ; i \omega_{l}\right) \equiv J_{0 \perp} \gamma\left(\boldsymbol{q} ; i \omega_{l}\right)^{2} .
$$

We can see directly from these expressions that the inter-layer Heisenberg interaction can be strongly enhanced by the strongly enhanced intra-layer spin fluctuations.

\section{Inter-layer Exchange Scattering Processes}

When we consider the spin fluctuating proceeses driven by the strongly enhanced inter-layer interaction, we should bear in mind that since in our treatment we neglect the inter-layer carrier hopping, the electron-hole pair bubble laid across adjacent layers doesn't exist. Therefore the most dominant process driven by the inter-layer interction is the electron-hole exchange scattering where an electron and a hole run in different layers. This kinds of processes are expected to develop dramatically just near the antiferromagnetic phase boundary and modify the simple RPA susceptibility. These scattering processes are described in terms of the T-matrices defined by

$$
\mathcal{T}_{\perp}^{\mu \nu, \lambda \rho}\left(k, k^{\prime} ; q\right)=\int_{0}^{1 / T} d \tau e^{i \omega_{l} \tau}<T_{\tau}\left[c_{1, \boldsymbol{k}^{\prime}, \mu}^{\dagger}(\tau) c_{1, \boldsymbol{k}^{\prime}+\boldsymbol{q}, \nu}(\tau) c_{2, \boldsymbol{k}+\boldsymbol{q}, \rho}^{\dagger}(0) c_{2, \boldsymbol{k}, \lambda}(0)\right]>,
$$

where $\mu, \nu, \lambda, \rho=\uparrow$ or $\downarrow$. 


\subsection{Scattering T-matrices}

The general expression (33) gives spin dependent T-matrices,

$$
\left\{\begin{array}{l}
\mathcal{T}_{\perp}^{\uparrow \downarrow, \uparrow \downarrow}(k, k ; q)=\mathcal{T}_{\perp}^{\downarrow \uparrow, \downarrow \uparrow}(k, k ; q), \\
\mathcal{T}_{\perp}^{\uparrow \uparrow, \uparrow \uparrow}\left(k, k^{\prime} ; q\right)=\mathcal{T}_{\perp}^{\downarrow \downarrow, \downarrow \downarrow}\left(k, k^{\prime} ; q\right), \\
\mathcal{T}_{\perp}^{\uparrow \downarrow, \downarrow \uparrow}\left(k, k^{\prime} ; q\right)=\mathcal{T}_{\perp}^{\downarrow \uparrow, \uparrow \downarrow}\left(k, k^{\prime} ; q\right) .
\end{array}\right.
$$

To clarify the nature of these spin dependent scattering processes, let us decompose them into the singlet and triplet channel. Since in our case T-matrices must satisfy the spin rotational symmetry relation, the scattering vertex can be written in a operator form in the spin space,

$$
\hat{\mathcal{T}}_{\perp}=\mathcal{T}_{\perp \rho}+\mathcal{T}_{\perp \sigma} \boldsymbol{\sigma}_{1} \cdot \boldsymbol{\sigma}_{2}
$$

where $\boldsymbol{\sigma}_{1}$ and $\boldsymbol{\sigma}_{2}$ denote the Pauli matrices that represent spin on the layer-1 and layer- 2 respectively. Then $\boldsymbol{\sigma}_{1} \cdot \boldsymbol{\sigma}_{2}$ has eigen values 1 and -3 that correspond to the triplet and singlet pair respectively. We can decompose $\mathcal{T}_{\perp}$ into the singlet and triplet channel as

$$
\left\{\begin{array}{c}
\mathcal{T}_{\perp}^{\text {triplet }}=\mathcal{T}_{\perp \rho}+\mathcal{T}_{\perp \sigma}, \\
\mathcal{T}_{\perp}^{\text {singlet }}=\mathcal{T}_{\perp \rho}-3 \mathcal{T}_{\perp \sigma} .
\end{array}\right.
$$

Thus we get

$$
\hat{\mathcal{T}}_{\perp}=\frac{\mathcal{T}_{\perp}^{\text {singlet }}+3 \mathcal{T}_{\perp}^{\text {triplet }}}{4}+\frac{\mathcal{T}_{\perp}^{\text {triplet }}-\mathcal{T}_{\perp}^{\text {singlet }}}{4} \sigma_{1} \cdot \sigma_{2}
$$

On the other hand, by noting $\boldsymbol{\sigma}_{1} \cdot \boldsymbol{\sigma}_{2}=2\left(\sigma_{1}^{+} \sigma_{2}^{-}+\sigma_{1}^{-} \sigma_{2}^{+}\right)+\sigma_{1}^{z} \sigma_{2}^{z}$, we get

$$
\left\{\begin{array}{c}
\mathcal{T}_{\perp}^{\uparrow \downarrow, \uparrow \downarrow}=\frac{1}{2}\left(\mathcal{T}_{\perp}^{\text {singlet }}+\mathcal{T}_{\perp}^{\text {triplet }}\right), \\
\mathcal{T}_{\perp}^{\uparrow \uparrow, \uparrow \uparrow}=\mathcal{T}_{\perp}^{\text {triplet }}, \\
\mathcal{T}_{\perp}^{\uparrow \downarrow, \downarrow \uparrow}=\frac{1}{2}\left(\mathcal{T}_{\perp}^{\text {triplet }}-\mathcal{T}_{\perp}^{\text {singlet }}\right) .
\end{array}\right.
$$

From these equations we can directly check the spin rotational invariance relation

$$
\mathcal{T}_{\perp}^{\uparrow \uparrow, \uparrow \uparrow}-\mathcal{T}_{\perp}^{\uparrow \downarrow, \downarrow \uparrow}=\mathcal{T}_{\perp}^{\uparrow \downarrow, \uparrow \downarrow}
$$

Finally we can combine $\mathcal{T}_{\perp}^{\text {singlet }}$ and $\mathcal{T}_{\perp}^{\text {triplet }}$ with $\mathcal{T}_{\perp}^{\mu \nu, \lambda \rho}$ like

$$
\begin{aligned}
& \mathcal{T}_{\perp}^{\text {singlet }}=\mathcal{T}^{\uparrow \downarrow, \uparrow \downarrow}-\mathcal{T}^{\uparrow \downarrow, \downarrow \uparrow}, \\
& \mathcal{T}_{\perp}^{\text {triplet }}=\mathcal{T}^{\uparrow \uparrow, \uparrow \uparrow} .
\end{aligned}
$$

In Fig. 5 we show the diagrammatic representation for the particel-hole ladder processes where the $n$-th order ladders are shown. As is shown in Fig.5(a), the type-C 
process produces $\mathcal{T}_{\perp(n)}^{\uparrow \downarrow, \uparrow \downarrow}(k, k ; q)$. As shown in Fig.5(b), the type-A and type-B process produce $\mathcal{T}_{\perp(n)}^{\uparrow \uparrow, \uparrow \uparrow}\left(k, k^{\prime} ; q\right)$ and $\mathcal{T}_{\perp(n)}^{\uparrow \downarrow, \downarrow \uparrow}\left(k, k^{\prime} ; q\right)$. In $\mathcal{T}_{\perp}^{\uparrow \uparrow, \uparrow \uparrow}(q)$, the initial spins of electron and hole are not flipped in the final state. On the other hand in $\mathcal{T}_{\perp}^{\uparrow \downarrow, \downarrow \uparrow}\left(k, k^{\prime} ; q\right)$, the initial spins are flipped in the final state. We note that although $\mathcal{T}_{\perp}^{\uparrow \downarrow, \uparrow \downarrow}$ includes only one scattering channel (type-C scattering) in all the intermediate states, $\mathcal{T}_{\perp}^{\uparrow \uparrow, \uparrow \uparrow}$ and $\mathcal{T}_{\perp}^{\uparrow \downarrow, \downarrow \uparrow}$ include two different scattering channels (type-A and type-B scattering) in all the possible ways. As a result the number of possible diagrams corersponding to Fig.6(b) increases more and more as the order of the diagram increases.

\section{$4.2 \mathcal{T}_{\perp}^{\uparrow \downarrow, \uparrow \downarrow}$}

We first consider $\mathcal{T}_{\perp}^{\uparrow \downarrow, \uparrow \downarrow}\left(k, k^{\prime} ; q\right)$. For example the 3rd order contribution can be explicitly written by

$\mathcal{T}_{\perp(3)}^{\uparrow \downarrow, \uparrow \downarrow}\left(k, k^{\prime} ; q\right)=-\frac{1}{64} \sum_{k_{1}, k_{2}} J_{\perp}\left(k-k_{1}\right) J_{\perp}\left(k_{1}-k_{2}\right) J_{\perp}\left(k_{2}-k^{\prime}\right) \mathcal{G}_{0}\left(k_{1}\right) \mathcal{G}_{0}\left(k_{1}+q\right) \mathcal{G}_{0}\left(k_{2}\right) \mathcal{G}_{0}\left(k_{2}+q\right)$.

To proceed with our analysis, we need two approximations. First we neglect the frequency dependence of the interaction and replace $J_{\perp}(q)$ with the most dominant contribution $J_{\perp}(\boldsymbol{q}) \equiv J_{\perp}\left(\boldsymbol{q} ; i \omega_{l}=0\right)$. Then we can perform the matsubara summations with regard to $k_{1}$ and $k_{2}$ in (42) and get

$$
\mathcal{T}_{\perp(3)}^{\uparrow \downarrow, \uparrow \downarrow}\left(k, k^{\prime} ; q\right)=-\frac{1}{64} \sum_{\boldsymbol{k}_{1}, \boldsymbol{k}_{2}} J_{\perp}\left(\boldsymbol{k}-\boldsymbol{k}_{1}\right) \chi_{\boldsymbol{k}_{1}}(q ; 0) J_{\perp}\left(\boldsymbol{k}_{1}-\boldsymbol{k}_{2}\right) \chi_{\boldsymbol{k}_{2}}(q ; 0) J_{\perp}\left(\boldsymbol{k}_{2}-\boldsymbol{k}^{\prime}\right)
$$

Now we introduced the quantity $\chi_{\boldsymbol{k}}(q ; Q)$ by

$$
\chi_{\boldsymbol{k}}(q ; Q) \equiv-T \sum_{\varepsilon_{n}} \mathcal{G}_{0}(k+q) \mathcal{G}_{0}(k+Q)=-\frac{1}{2} \frac{\tanh \frac{\xi_{\boldsymbol{k}+\boldsymbol{q}}}{2 T}-\tanh \frac{\xi_{\boldsymbol{k}+\boldsymbol{Q}}}{2 T}}{i \omega_{l}-i \Omega_{m}-\eta_{\boldsymbol{k}}(\boldsymbol{q} ; \boldsymbol{Q})} .
$$

We note that $J_{\perp}(\boldsymbol{q})$ has a broad maximum around the antiferromagnetic vector $\boldsymbol{q}^{*}=$ $(\pi / q, \pi / a)$. Furthermore the internal wavenumber $\boldsymbol{k}$ and $\boldsymbol{k}^{\prime}$ become important only when they satisfy the condition $\boldsymbol{k}-\boldsymbol{k}^{\prime} \sim \mathbf{0}$ (forward scattering) or $\boldsymbol{k}-\boldsymbol{k}^{\prime} \sim \boldsymbol{q}^{*}$ (backward scattering). The latter process is strongly driven by the antiferromagnetic spin fluctuations. We can see that the 3rd order process is enhanced only in the case of backward scattering, since $\boldsymbol{k}-\boldsymbol{k}_{1} \sim \boldsymbol{q}^{*}$ and $\boldsymbol{k}_{1}-\boldsymbol{k}_{2} \sim \boldsymbol{q}^{*}$ give constraint on $\boldsymbol{k}^{\prime}$ as $\boldsymbol{k}^{\prime} \sim \boldsymbol{k}+\boldsymbol{q}^{*}$.

This consideration gives us important results: the ladders with odd number $J_{\perp}(\boldsymbol{q})$ give dominant contribution to backward scattering processes, while the ladders with even number $J_{\perp}(\boldsymbol{q})$ give dominant contribution to forward scattering processes. This situations are shown in Fig. 6 Keeping this constraints in mind, we replace $J_{\perp}(\boldsymbol{q})$ with the averaged one 1

$$
\mathcal{J}_{\perp}(T)=\sum_{\boldsymbol{q}} J_{\perp}\left(\boldsymbol{q}, i \omega_{l}=0\right)=J_{0 \perp} \sum_{\boldsymbol{q}}\left\{\gamma\left(\boldsymbol{q}, i \omega_{l}=0\right)\right\}^{2}
$$

\footnotetext{
${ }^{1}$ To replace the non-separable momentum dependent interaction with its averaged value is in-
} 
which depends on the temperature through $\chi_{0}(\boldsymbol{q} ; 0)$ in the Stoner factors. This procedure is our second approximation. Now $\mathcal{T}_{\perp(3)}^{\uparrow \downarrow, \uparrow \downarrow}\left(k, k^{\prime} ; q\right)$ can be approximated simply by

$$
\mathcal{T}_{\perp(3)}^{\uparrow \downarrow, \uparrow \downarrow}\left(k, k^{\prime} ; q\right)=-\left\{\frac{\mathcal{J}_{\perp}(T)}{4}\right\}^{3} \chi_{0}(q)^{2},
$$

where $\chi_{0}(q)=\sum_{\boldsymbol{k}} \chi_{\boldsymbol{k}}(q, 0)$. At this stage T-matrix depends only on $q$. Thus from now on we simply denote the T-matrix as $\mathcal{T}_{\perp}^{\uparrow \downarrow, \uparrow \downarrow}(q)$.

Genelarizing the aformentioned procedure, we can get the T-matrixes for forward and backward process as

$$
\begin{gathered}
\mathcal{T}_{\perp \downarrow W W}^{\uparrow \downarrow, \downarrow}(q)=\frac{\left\{\frac{\mathcal{J}_{\perp}(T)}{4}\right\}^{2} \chi_{0}(q)}{1-\left\{\frac{\mathcal{J}_{\perp}(T)}{4} \chi_{0}(q)\right\}^{2}}, \\
\mathcal{T}_{\perp B W W}^{\uparrow \downarrow, \downarrow}(q)=-\frac{\left\{\frac{\mathcal{J}_{\perp}(T)}{4}\right\}^{3} \chi_{0}(q)^{2}}{1-\left\{\frac{\mathcal{J}_{\perp}(T)}{4} \chi_{0}(q)\right\}^{2}},
\end{gathered}
$$

where FW and BW denote the case of forward and backward process respectively.

\section{3 $\mathcal{T}_{\perp}^{\uparrow \uparrow, \uparrow \uparrow}$ and $\mathcal{T}_{\perp}^{\uparrow \downarrow, \downarrow \uparrow}$}

Next we consider $\mathcal{T}_{\perp(n)}^{\uparrow \uparrow, \uparrow}$ and $\mathcal{T}_{\perp(n)}^{\uparrow \downarrow, \downarrow \uparrow}$. To get explicit expressions for them, let us introduce the auxiliary spin-independent T-matrix $\mathcal{T}_{\perp(n)}$ that is defined in Fig.5(c). In $\mathcal{T}_{\perp(n)}$, all the inter-plane interactions contained in the ladder are type-B interaction. In reality $\mathcal{T}_{\perp(n)}^{\uparrow \uparrow, \uparrow \uparrow}$ and $\mathcal{T}_{\perp(n)}^{\uparrow \perp, \downarrow \uparrow}$ contain type- $\mathrm{A}$ and type-B interactions in all the possible ways. Then $n$-th order ladder $\mathcal{T}_{\perp(n)}^{\uparrow \uparrow, \uparrow \uparrow}$ and $\mathcal{T}_{\perp(n)}^{\uparrow \downarrow, \downarrow \uparrow}$ can simply be related to corresponding $\mathcal{T}_{\perp(n)}$ through combinatrics factor as

$$
\begin{aligned}
& \mathcal{T}_{\perp(n)}^{\uparrow \uparrow, \uparrow \uparrow}=a_{n} \mathcal{T}_{\perp(n)} \\
& \mathcal{T}_{\perp(n)}^{\uparrow \downarrow, \downarrow \uparrow}=b_{n} \mathcal{T}_{\perp(n)}
\end{aligned}
$$

where

$$
\begin{aligned}
& a_{n}=\sum_{i=0}^{\left[\frac{n}{2}\right]} 2^{2 i}{ }_{n} C_{2 i}, \\
& b_{n}=\sum_{i=0}^{\left[\frac{n}{2}-\frac{1}{2}\right]} 2^{2 i+1}{ }_{n} C_{2 i+1} .
\end{aligned}
$$

evitable to solve the Bethe-Salpeter equation in a closed form. In the case of Coulomb gas, the dielectric screening removes the singularity and as a result this approximation may work well. Wolff discussed the spin susceptibility of electron gas in this manner 53.

In the present problem, the spin-fluctuation mediated interaction $J_{\perp}(q)$ has short range nature and doesn't have any singularity in a paramagnetic phase. Therefore in the present case this approximation can be justified. 
Here ${ }_{n} C_{m}=n ! /[(m-n) ! m !]$ is a binomial coefficient.

The above relations have already been discussed in Ref. [54]. Now, for example, we derive the formula (49). In this case $n$-th order term of $\mathcal{T}_{\perp(n)}^{\uparrow \uparrow, \uparrow}$ can include the even number spin-flipping (Type-A) scattering. Then all the other scatterings are the type-B scattering. We consider the case when there are $2 i(i=0,1, \cdots)$ type-A processses. Then there are $n-2 i$ Type-B vertices in the $n$-th order ladder. Since the type-A vertex gives the facor $J_{0 \perp} / 2$ and the type-B vertex gives the facor $J_{0 \perp} / 4$, if we replace all the type-A vertices simply with the Type-B vertices to get $\mathcal{T}_{\perp(n)}$, there appear the factor $2^{2 i}$. Furthermore, concerning the location of the type-A interactions in a ladder there are ${ }_{n} C_{2 i}$ ways. As a result $\mathcal{T}_{\perp(n)}^{\uparrow \uparrow, \uparrow}$ is related with $\mathcal{T}_{\perp(n)}$ through a factor $\sum_{i=0}^{\left[\frac{n}{2}\right]} 2^{2 i}{ }_{n} C_{2 i}$.

Now we notice that $n$-th order prefactors satisfy the simple relations

$$
a_{n}+b_{n}=(1+2)^{n}=3^{n},
$$

and

$$
a_{n}-b_{n}=(1-2)^{n}=(-1)^{n} .
$$

We can get actual feeling for these relations by writing down concrete values of multiplication factors

$$
\left\{\begin{array}{l}
a_{2}=5 \\
b_{2}=4
\end{array},\left\{\begin{array}{l}
a_{3}=13 \\
b_{3}=14
\end{array},\left\{\begin{array}{l}
a_{4}=41 \\
b_{4}=40
\end{array},\left\{\begin{array}{l}
a_{5}=121 \\
b_{5}=122
\end{array}, \cdots .\right.\right.\right.\right.
$$

Using the relation, (53), we can obtain

$$
\mathcal{T}_{\perp(n)}^{\uparrow \uparrow, \uparrow \uparrow}+\mathcal{T}_{\perp(n)}^{\uparrow \downarrow, \downarrow \uparrow}=3^{n} \mathcal{T}_{\perp(n)}
$$

Therefore we can get the explicit result for $\mathcal{T}_{\perp}^{\uparrow \uparrow, \uparrow \uparrow}+\mathcal{T}_{\perp}^{\uparrow \downarrow, \downarrow \uparrow}$. In the case of forward process, dominant contributions arise from

$$
\mathcal{T}_{\perp F W}^{\uparrow, \uparrow \uparrow}+\mathcal{T}_{\perp F W}^{\uparrow \downarrow, \downarrow \uparrow}=\frac{\left\{\frac{3}{4} \mathcal{J}_{\perp}(T)\right\}^{2} \chi_{0}(q)}{1-\left\{\frac{3}{4} \mathcal{J}_{\perp}(T) \chi_{0}(q)\right\}^{2}},
$$

while in the case of backward process, dominant contributions arise from

$$
\mathcal{T}_{\perp B W}^{\uparrow \uparrow, \uparrow \uparrow}+\mathcal{T}_{\perp B W}^{\uparrow \perp, \perp \uparrow}=\frac{\left\{\frac{3}{4} \mathcal{J}_{\perp}(T)\right\}^{3} \chi_{0}(q)^{2}}{1-\left\{\frac{3}{4} \mathcal{J}_{\perp}(T) \chi_{0}(q)\right\}^{2}} .
$$

Next we go back to the relation (54) that gives the simple relation

$$
\mathcal{T}_{\perp(n)}^{\uparrow \uparrow, \uparrow \uparrow}-\mathcal{T}_{\perp(n)}^{\uparrow \downarrow, \downarrow \uparrow}=(-1)^{n} \mathcal{T}_{\perp(n)}
$$

Now we can notice that

$$
(-1)^{n} \mathcal{T}_{\perp(n)}=\mathcal{T}_{\perp(n)}^{\uparrow \downarrow, \uparrow \downarrow}
$$


Therefore the relation (54) directly leads to the spin-rotational symmetry relation

$$
\mathcal{T}_{\perp}^{\uparrow \uparrow, \uparrow \uparrow}-\mathcal{T}_{\perp}^{\uparrow \downarrow, \downarrow \uparrow}=\mathcal{T}_{\perp}^{\uparrow \downarrow, \uparrow \downarrow} .
$$

Thus we can confirm again that the present treatment doesn't violate the spin rotaional symmetry.

The relations (56), (57) and (60) enable us to get the explicit form of $\mathcal{T}_{\perp}^{\uparrow \uparrow, \uparrow \uparrow}$ and $\mathcal{T}_{\perp}^{\uparrow \downarrow, \downarrow \uparrow}$. We get

$$
\begin{aligned}
& \mathcal{T}_{\perp F W}^{\uparrow \uparrow, \uparrow \uparrow}(q)=\frac{1}{2}\left[\frac{\left\{\frac{3}{4} \mathcal{J}_{\perp}(T)\right\}^{2} \chi_{0}(q)}{1-\left\{\frac{3}{4} \mathcal{J}_{\perp}(T) \chi_{0}(q)\right\}^{2}}+\frac{\left\{\frac{1}{4} \mathcal{J}_{\perp}(T)\right\}^{2} \chi_{0}(q)}{1-\left\{\frac{1}{4} \mathcal{J}_{\perp}(T) \chi_{0}(q)\right\}^{2}}\right], \\
& \mathcal{T}_{\perp F W}^{\uparrow \downarrow, \downarrow \uparrow}(q)=\frac{1}{2}\left[\frac{\left\{\frac{3}{4} \mathcal{J}_{\perp}(T)\right\}^{2} \chi_{0}(q)}{1-\left\{\frac{3}{4} \mathcal{J}_{\perp}(T) \chi_{0}(q)\right\}^{2}}-\frac{\left\{\frac{1}{4} \mathcal{J}_{\perp}(T)\right\}^{2} \chi_{0}(q)}{1-\left\{\frac{1}{4} \mathcal{J}_{\perp}(T) \chi_{0}(q)\right\}^{2}}\right],
\end{aligned}
$$

for forward process and

$$
\begin{aligned}
& \mathcal{T}_{\perp B W}^{\uparrow \uparrow, \uparrow \uparrow}(q)=\frac{1}{2}\left[\frac{\left\{\frac{3}{4} \mathcal{J}_{\perp}(T)\right\}^{3} \chi_{0}(q)^{2}}{1-\left\{\frac{3}{4} \mathcal{J}_{\perp}(T) \chi_{0}(q)\right\}^{2}}-\frac{\left\{\frac{1}{4} \mathcal{J}_{\perp}(T)\right\}^{3} \chi_{0}(q)^{2}}{1-\left\{\frac{1}{4} \mathcal{J}_{\perp}(T) \chi_{0}(q)\right\}^{2}}\right], \\
& \mathcal{T}_{\perp B W}^{\uparrow \perp, \downarrow \uparrow}(q)=\frac{1}{2}\left[\frac{\left\{\frac{3}{4} \mathcal{J}_{\perp}(T)\right\}^{3} \chi_{0}(q)^{2}}{1-\left\{\frac{3}{4} \mathcal{J}_{\perp}(T) \chi_{0}(q)\right\}^{2}}+\frac{\left\{\frac{1}{4} \mathcal{J}_{\perp}(T)\right\}^{3} \chi_{0}(q)^{2}}{1-\left\{\frac{1}{4} \mathcal{J}_{\perp}(T) \chi_{0}(q)\right\}^{2}}\right],
\end{aligned}
$$

for backward process.

\section{4 $\mathcal{T}_{\perp}^{\text {singlet }}$ and $\mathcal{T}_{\perp}^{\text {triplet }}$}

By using (40), (41) and (61) (64), we can get the explicit form of the T-matrices

in the singlet and triplet channel in the case of the forward and backward process as

$$
\begin{aligned}
& \mathcal{T}_{\perp F W}^{\text {singlet }}(q)=\frac{3}{2} \frac{\left\{\frac{1}{4} \mathcal{J}_{\perp}(T)\right\}^{2} \chi_{0}(q)}{1-\left\{\frac{1}{4} \mathcal{J}_{\perp}(T) \chi_{0}(q)\right\}^{2}}-\frac{1}{2} \frac{\left\{\frac{3}{4} \mathcal{J}_{\perp}(T)\right\}^{2} \chi_{0}(q)}{1-\left\{\frac{3}{4} \mathcal{J}_{\perp}(T) \chi_{0}(q)\right\}^{2}}, \\
& \mathcal{T}_{\perp F W}^{\text {triplet }}(q)=\frac{1}{2} \frac{\left\{\frac{3}{4} \mathcal{J}_{\perp}(T)\right\}^{2} \chi_{0}(q)}{1-\left\{\frac{3}{4} \mathcal{J}_{\perp}(T) \chi_{0}(q)\right\}^{2}}+\frac{1}{2} \frac{\left\{\frac{1}{4} \mathcal{J}_{\perp}(T)\right\}^{2} \chi_{0}(q)}{1-\left\{\frac{1}{4} \mathcal{J}_{\perp}(T) \chi_{0}(q)\right\}^{2}},
\end{aligned}
$$

and

$$
\begin{gathered}
\mathcal{T}_{\perp B W}^{\text {singlet }}(q)=-\frac{3}{2} \frac{\left\{\frac{1}{4} \mathcal{J}_{\perp}(T)\right\}^{3} \chi_{0}(q)^{2}}{1-\left\{\frac{1}{4} \mathcal{J}_{\perp}(T) \chi_{0}(q)\right\}^{2}}-\frac{1}{2} \frac{\left\{\frac{3}{4} \mathcal{J}_{\perp}(T)\right\}^{3} \chi_{0}(q)^{2}}{1-\left\{\frac{3}{4} \mathcal{J}_{\perp}(T) \chi_{0}(q)\right\}^{2}} \\
\mathcal{T}_{\perp B W}^{\text {triplet }}(q)=\frac{1}{2} \frac{\left\{\frac{3}{4} \mathcal{J}_{\perp}(T)\right\}^{3} \chi_{0}(q)^{2}}{1-\left\{\frac{3}{4} \mathcal{J}_{\perp}(T) \chi_{0}(q)\right\}^{2}}-\frac{1}{2} \frac{\left\{\frac{1}{4} \mathcal{J}_{\perp}(T)\right\}^{3} \chi_{0}(q)^{2}}{1-\left\{\frac{1}{4} \mathcal{J}_{\perp}(T) \chi_{0}(q)\right\}^{2}}
\end{gathered}
$$


In Fig. 7, we show the behavior of the singlet and triplet T-matrices as a function of the dimensionless quantity, $\mathcal{J}_{\perp}(T) \chi_{0}(q) / 4$. It follows that the inter-layer exchange scattering processes give rise to the strong attraction for the spin singlet pair and the strong repulsion for the spin triplet pair. Thus we can say that just near the intra-layer antiferromagnetic phase boundary the inter-layer singlet pair formation is strongly favored while the triplet pair formation is strongly prohibited. This tendency mainly comes from the dramatically enhanced repulsion between the inter-layer parallel spin pair, $\mathcal{T}_{\perp}^{\uparrow \uparrow, \uparrow \uparrow}(q)$ and $\mathcal{T}_{\perp}^{\uparrow \downarrow, \downarrow \uparrow}(q)$. We can expect that the strong repulsion for inter-layer parallel spin pairs induces the screening effects for the intra- and inter-layer spin fluctuations. We investigate this screening effects in the next section.

\subsection{Averaged Inter-layer Interaction $\mathcal{J}_{\perp}(T)$}

It is should be noted that the Stoner factor sensitively depends on the magnitude of the intra-layer Hubbard interaction. Our proposal can be justified only when the Stoner factor becomes considerablly large. In Fig. 8. we show the numerical results for the averaged inter-layer interaction $\mathcal{J}_{\perp}$ for $U=3.25 t$. Here the half-filling corresponds to $\mu=-0.327 t$. We fixed the chemical potential to $\mu=-0.54 t$. By taking up the avarage, we can also check the non-divergence of the Stoner factor over the whole Brillouin zone. If the bare susceptibility touches the critical condition, $U \chi_{0}(q)=1$, at a certain temperature, $T_{\mathrm{SDW}}$, the RPA susceptibility diverges and the whole theoretical backgrounds break down.

As for the magnitude of $J_{0 \perp}$, we set $J_{0 \perp}=0.08 \mathrm{t}$. This magnitude of the bare inter-layre Heisenberg interaction corresponds to the result suggested in Ref. 52. The maximam of the bare susceptibility, $\chi_{0}(q)$, reach $0.26 / t$. Therefore, the divergence of $\mathcal{T}_{\perp}^{\text {singlet,triplet }}$ occurs at $0.75 \mathcal{J}_{\perp} \chi_{0}=1$ that corresponds to $0.75 \mathcal{J}_{\perp} \sim 3.8$. As we can see from the result of Fig. 8, the numerical vale of $0.75 \mathcal{J}_{\perp}$ can remain finite down to $T=0$ with the maximum value, $0.75 \mathcal{J}_{\perp} \sim 3.9$. If we take larger magnitude of $U, \mathcal{J}_{\perp}$ becomes divergent at unreallistically high temperature, $T_{S D W}$.

\section{Contributions of Exchange Scattering Processes to Diagonal and Off-diagonal Susceptibility}

Now we consider explicitly how inter-layer exchange scattering processees affect the irreducible diagonal- and off-diagonal susceptibilities. Within the present scheme, the inter-layer exchange processes cannot be detected directly through experimental probe because in our model there is no inter-layer direct carrier hopping. Instead these processes are inserted into the irreducible polarization. In Fig. 9 we show the contributions from exchange scattering T-matrices to the transverse susceptibility up to the 2nd order. Among them (a),(b), (c) and (d) contribute to the diagonal counterpart of the transverse susceptibility, $\Delta \chi_{/ /}^{+-}$, although (e),(f), and (g) contribute to the off-diagonal counterpart, $\Delta \chi_{\perp}^{+-}$. In the appendix we shall discuss the longitudi- 
nal counterpart, $\Delta \chi_{/ /}^{z z}$ and $\Delta \chi_{\perp}^{z z}$, and prove the spin rotational invariance relation. As we shall discuss in the next section, we can notice that the neutron scattering experiments detect all diagrams in Fig. 9, while the NMR longitudinal relaxation experiments detect only diagram (a),(b),(c), and (d). Furthermore all diagrams contribute to the NMR spin echo decay rate. We note that in each diagram the forward and backward T-matrices are inserted correspondong to the characteristic momentum transfer. Thus we get the expressions for the correction terms to the diagonal counterpart as

$$
\Delta \chi_{/ /}^{+-}=\Delta \chi_{/ /, \mathrm{a}}^{+-}+\Delta \chi_{/ /, \mathrm{b}}^{+-}+\Delta \chi_{/ /, \mathrm{c}}^{+-}+\Delta \chi_{/ /, \mathrm{d}}^{+-},
$$

where

$$
\begin{aligned}
& \Delta \chi_{/ /, \mathrm{a}}^{+-}=-\gamma(q)^{2} T \sum_{\Omega_{m}} \sum_{\boldsymbol{Q}} \mathcal{T}_{\perp F W}^{\uparrow \uparrow, \uparrow \uparrow}(Q) \mathcal{L}^{(1)}(q, Q), \\
& \Delta \chi_{/ /, \mathrm{b}}^{+-}=-\gamma(q)^{2} T \sum_{\Omega_{m}} \sum_{\boldsymbol{Q}} \mathcal{T}_{\perp F W}^{\uparrow \downarrow, \uparrow \downarrow}(Q) \mathcal{L}^{(1)}(q, Q), \\
& \Delta \chi_{/ /, \mathrm{c}}^{+-}=\gamma(q)^{2} T \sum_{\Omega_{m}} \sum_{\boldsymbol{Q}}\left\{\mathcal{T}_{\perp F W}^{\uparrow \uparrow, \uparrow \uparrow}(Q) \mathcal{T}_{\perp F W}^{\uparrow \downarrow, \uparrow \downarrow}(Q+q)+\mathcal{T}_{\perp B W}^{\uparrow \uparrow, \uparrow \uparrow}(Q) \mathcal{T}_{\perp B W}^{\uparrow \perp, \uparrow \downarrow}(Q+q)\right\} \\
& \times\left\{\mathcal{L}^{(3)}(q, Q)\right\}^{2} \\
& \Delta \chi_{/ /, \mathrm{d}}^{+-}=\gamma(q)^{2} T \sum_{\Omega_{m}} \sum_{\boldsymbol{Q}}\left\{\mathcal{T}_{\perp F W}^{\uparrow \downarrow, \downarrow \uparrow}(Q) \mathcal{T}_{\perp F W}^{\downarrow \downarrow, \downarrow \downarrow}(Q+q)+\mathcal{T}_{\perp B W}^{\uparrow \downarrow, \downarrow \uparrow}(Q) \mathcal{T}_{\perp B W}^{\downarrow \downarrow, \downarrow \downarrow \downarrow}(Q+q)\right\} \\
& \times\left\{\mathcal{L}^{(3)}(q, Q)\right\}^{2}
\end{aligned}
$$

Each indices a, b, c, ... represent the corresponding diagrams in Fig. 9. Furthermore the correction terms to the off-diagonal counterpart are given by

$$
\Delta \chi_{\perp}^{+-}=\Delta \chi_{\perp, \mathrm{e}}^{+-}+\Delta \chi_{\perp, \mathrm{f}}^{+-}+\Delta \chi_{\perp, \mathrm{g}}^{+-}
$$

where

$$
\begin{aligned}
& \Delta \chi_{\perp, \mathrm{e}}^{+-}=-\gamma(q)^{2} T \sum_{\Omega_{m}} \sum_{\boldsymbol{Q}} \mathcal{T}_{\perp B W}^{\uparrow \downarrow, \downarrow \uparrow}(Q) \mathcal{L}^{(2)}(q, Q), \\
& \Delta \chi_{\perp, \mathrm{f}}^{+-}=\gamma(q)^{2} T \sum_{\Omega_{m}} \sum_{Q}\left[\mathcal{T}_{\perp F W}^{\uparrow \downarrow, \uparrow \downarrow}(Q) \mathcal{T}_{\perp B W}^{\uparrow \downarrow, \downarrow \uparrow}(Q+q)+\mathcal{T}_{\perp B W}^{\uparrow \downarrow, \uparrow \downarrow}(Q) \mathcal{T}_{\perp F W}^{\uparrow \downarrow, \downarrow \uparrow}(Q+q)\right. \\
& \left.+\mathcal{T}_{\perp F W}^{\uparrow \downarrow, \uparrow \downarrow}(Q) \mathcal{T}_{\perp B W}^{\uparrow \downarrow, \downarrow \uparrow}(Q+q)+\mathcal{T}_{\perp B W}^{\uparrow \downarrow, \uparrow \downarrow}(Q) \mathcal{T}_{\perp F W}^{\uparrow \downarrow, \downarrow \uparrow}(Q+q)\right] \mathcal{L}^{(3)}(q, Q) \mathcal{L}^{(3)}(-q,-Q), \\
& \Delta \chi_{\perp, g}^{+-}=\gamma(q)^{2} T \sum_{\Omega_{m}} \sum_{\boldsymbol{Q}}\left[\mathcal{T}_{\perp F W}^{\uparrow \downarrow, \downarrow \uparrow}(Q) \mathcal{T}_{\perp B W}^{\uparrow \downarrow, \uparrow \downarrow}(Q+q)+\mathcal{T}_{\perp B W}^{\uparrow \downarrow, \downarrow \uparrow}(Q) \mathcal{T}_{\perp F W}^{\uparrow \downarrow, \uparrow \downarrow}(Q+q)\right. \\
& \left.+\mathcal{T}_{\perp F W}^{\uparrow \downarrow, \downarrow \uparrow}(Q) \mathcal{T}_{\perp B W}^{\uparrow \downarrow, \uparrow \downarrow}(Q+q)+\mathcal{T}_{\perp B W}^{\uparrow \downarrow, \downarrow \uparrow}(Q) \mathcal{T}_{\perp F W}^{\uparrow \downarrow, \uparrow \downarrow}(Q+q)\right] \mathcal{L}^{(3)}(q, Q) \mathcal{L}^{(3)}(-q,-Q) .
\end{aligned}
$$

We note that in the diagram (c), (d), (f), and (g), forward and backward counterparts are combined to each other. The coupling functions are given by

$$
\mathcal{L}^{(1)}\left(\boldsymbol{q} ; i \omega_{l}, \boldsymbol{Q} ; i \Omega_{m}\right)=T \sum_{\varepsilon_{n}} \sum_{\boldsymbol{k}}\left\{\mathcal{G}_{0}(k)\right\}^{2} \mathcal{G}_{0}(k+q) \mathcal{G}_{0}(k+Q)
$$




$$
\begin{array}{r}
=T \sum_{\varepsilon_{n}} \sum_{\boldsymbol{k}} \frac{\mathcal{G}_{0}(k)-\mathcal{G}_{0}(k+q)}{i \omega_{l}-\xi_{\boldsymbol{k}+\boldsymbol{q}}+\xi_{\boldsymbol{k}}} \frac{\mathcal{G}_{0}(k)-\mathcal{G}_{0}(k+Q)}{i \Omega_{m}-\xi_{\boldsymbol{k}+\boldsymbol{Q}}+\xi_{\boldsymbol{k}}} \\
=\sum_{\boldsymbol{k}} \frac{-\frac{1}{4 T} \cosh ^{-2} \frac{\xi_{\boldsymbol{k}}}{2 T}+\chi_{\boldsymbol{k}}(0 ; Q)+\chi_{\boldsymbol{k}}(q ; 0)-\chi_{\boldsymbol{k}}(q ; Q)}{\left[i \omega_{l}-\xi_{\boldsymbol{k}+\boldsymbol{q}}+\xi_{\boldsymbol{k}}\right]\left[i \Omega_{m}-\xi_{\boldsymbol{k}+\boldsymbol{Q}}+\xi_{\boldsymbol{k}}\right]}, \\
\mathcal{L}^{(2)}\left(\boldsymbol{q} ; i \omega_{l}, \boldsymbol{Q} ; i \Omega_{m}\right)=T \sum_{\varepsilon_{n}} \sum_{\boldsymbol{k}} \mathcal{G}_{0}(k) \mathcal{G}_{0}(k+q) \mathcal{G}_{0}(k+Q) \mathcal{G}_{0}(k+Q+q) \\
=\frac{T}{N} \sum_{\varepsilon_{n}} \sum_{\boldsymbol{k}} \frac{\mathcal{G}_{0}(k)-\mathcal{G}_{0}(k+q)}{i \omega_{l}-\eta_{\boldsymbol{k}}(\boldsymbol{q}, 0)} \frac{\mathcal{G}_{0}(k+Q)-\mathcal{G}_{0}(k+q+Q)}{i \omega_{l}-\eta_{\boldsymbol{k}}(\boldsymbol{q}+\boldsymbol{Q}, \boldsymbol{Q})} \\
=\sum_{\boldsymbol{k}} \frac{-\chi_{\boldsymbol{k}}(0 ; Q)+\chi_{\boldsymbol{k}}(0 ; q+Q)+\chi_{\boldsymbol{k}}(q ; Q)-\chi_{\boldsymbol{k}}(q ; q+Q)}{\left[i \omega_{l}-\eta_{\boldsymbol{k}}(\boldsymbol{q}, 0)\right]\left[i \omega_{l}-\eta_{\boldsymbol{k}}(\boldsymbol{q}+\boldsymbol{Q}, \boldsymbol{Q})\right]},
\end{array}
$$

and

$$
\begin{array}{r}
\mathcal{L}^{(3)}\left(\boldsymbol{q} ; i \omega_{l}, \boldsymbol{Q} ; i \Omega_{m}\right)=T \sum_{\varepsilon_{n}} \sum_{\boldsymbol{k}} \mathcal{G}_{0}(k) \mathcal{G}_{0}(k+Q) \mathcal{G}(k+Q+q) \\
=T \sum_{\varepsilon_{n}} \sum_{\boldsymbol{k}} \frac{\mathcal{G}_{0}(k) \mathcal{G}_{0}(k+Q+q)-\mathcal{G}_{0}(k+Q) \mathcal{G}_{0}(k+Q+q)}{i \Omega_{m}-\eta_{\boldsymbol{k}}(\boldsymbol{Q}, 0)} \\
=\sum_{\boldsymbol{k}} \frac{-\chi_{\boldsymbol{k}}(0 ; q+Q)+\chi_{\boldsymbol{k}}(Q ; q+Q)}{i \Omega_{m}-\eta_{\boldsymbol{k}}(Q, 0)},
\end{array}
$$

where $\chi_{\boldsymbol{k}}(q ; Q)$ has already been defined by (44) and $\eta_{\boldsymbol{k}}(\boldsymbol{q}, \boldsymbol{Q})=\xi_{\boldsymbol{k}+\boldsymbol{q}}-\xi_{\boldsymbol{k}+\boldsymbol{Q}}$. In the above expressions, $q=\left(\boldsymbol{q}, i \omega_{l}\right), Q=\left(\boldsymbol{Q}, i \Omega_{m}\right)$ where $\omega_{l}$ and $\Omega_{m}$ denote bosonic frequencies.

In the terminology of superconducting fluctuation, (a), (b), and (e) correspond to Maki-Thompson diagram [56], although (c), (d), (f), and (g) correspond to AslamazovLarkin diagram 55. Maki-Thompson terms represent the coupling of spin fluctuations via the exchange scattering processes with the internal momentum transfer $\boldsymbol{Q}$, although Aslamazov-Larkin term represent the coupling of the fluctuations via the processes with the internal momentum transfer $\boldsymbol{Q}$ and $\boldsymbol{Q}+\boldsymbol{q}$. Therefore in the case of the antiferromagnetic spin fluctuations, at the commensurate momentum $\boldsymbol{q}=\boldsymbol{q}^{*}=(\pi / a, \pi / a)$, the coupling functions in the Aslamazov-Larkin terms are severely reduced. Moreover in our case Aslamazov-Larkin terms inevitablly include $\mathcal{T}_{\perp}^{\uparrow \downarrow, \uparrow \downarrow}$ that is only weakly enhanced, and therefore can safely be discarded.

Based on the above consideration, we can say that the dominant contribution to the diagonal susceptibility arises from the diagram Fig. 9(a), i.e.

$$
\Delta \chi_{/ /}^{+-} \sim \Delta \chi_{/ /, \mathrm{a}}^{+-}
$$

Furthermore the most dominant contribution to the off-diagonal counterpart arises form the diagram Fig. 9(e), i.e.

$$
\Delta \chi_{\perp}^{+-} \sim \Delta \chi_{\perp, \mathrm{e}}^{+-}
$$


Since the dominant contribution of T-matrices, $\mathcal{T}_{\perp F W, B W}^{\mu \nu, \lambda \rho}(Q)$, arises from the terms with its external frequency $\Omega_{m}=0$, from now on we put $\Omega_{m}=0$ in $Q$. Moreover the dominant contribution of $\mathcal{T}_{\perp F W, B W}^{\mu \nu, \lambda \rho}\left(\Omega_{m}=0, \boldsymbol{Q}\right)$ in $(74)$ and $(\sqrt{75})$ arises form the term with $\boldsymbol{q} \sim \boldsymbol{Q} \sim \boldsymbol{q}^{*}$. Thus

$$
\Delta \chi_{/ /}^{+-}\left(\boldsymbol{q}^{*} ; i \omega_{l}\right) \sim-T \gamma\left(\boldsymbol{q}^{*} ; i \omega_{l}\right)^{2} \mathcal{T}_{\perp F W}^{\uparrow \uparrow, \uparrow \uparrow}\left(\boldsymbol{q}^{*} ; i \Omega_{m}=0\right) \mathcal{L}^{(1)}\left(\boldsymbol{q}^{*} ; i \omega_{l} ; \boldsymbol{q}^{*} ; i \Omega_{m}=0\right),
$$

and

$$
\Delta \chi_{\perp}^{+-}\left(\boldsymbol{q} ; i \omega_{l}\right) \sim-T \gamma\left(\boldsymbol{q} ; i \omega_{l}\right)^{2} \mathcal{T}_{\perp B W}^{\uparrow \downarrow, \downarrow \uparrow}\left(\boldsymbol{q}^{*} ; i \Omega_{m}=0\right) \mathcal{L}^{(2)}\left(\boldsymbol{q}^{*} ; i \Omega_{l} ; \boldsymbol{q}^{*} ; i \Omega_{m}=0\right) .
$$

Then we get the explicit expression for coupling functions as

$$
\begin{aligned}
\mathcal{L}^{(1)}\left(\boldsymbol{q}^{*} ; i \omega_{l} ; \boldsymbol{q}^{*} ; i \Omega_{m}=0\right) & =\sum_{\boldsymbol{k}}\left[\frac{-\frac{1}{2 T} \cosh ^{-2} \frac{\xi \boldsymbol{k}}{2 T}}{\omega_{l}^{2}+\eta_{\boldsymbol{k}}\left(\boldsymbol{q}^{*} ; 0\right)^{2}}+2 \frac{\eta_{\boldsymbol{k}}\left(\boldsymbol{q}^{*} ; 0\right) \Lambda_{\boldsymbol{k}}\left(\boldsymbol{q}^{*} ; 0\right)}{\left\{\omega_{l}^{2}+\eta_{\boldsymbol{k}}\left(\boldsymbol{q}^{*} ; 0\right)^{2}\right\}^{2}}\right] \\
\mathcal{L}^{(2)}\left(\boldsymbol{q}^{*} ; i \omega_{l} ; \boldsymbol{q}^{*} ; i \Omega_{m}=0\right) & =\frac{1}{2} \sum_{\boldsymbol{k}} \frac{1}{\eta_{\boldsymbol{k}}\left(\boldsymbol{q}^{*} ; 0\right)} \frac{\Lambda_{\boldsymbol{k}}\left(\boldsymbol{q}^{*} ; 0\right)}{\omega_{l}^{2}+\eta_{\boldsymbol{k}}\left(\boldsymbol{q}^{*}, 0\right)^{2}} \\
\mathcal{L}^{(3)}\left(\boldsymbol{q}^{*} ; i \omega_{l} ; \boldsymbol{q}^{*} ; i \Omega_{m}=0\right) & =-\frac{1}{2} \sum_{\boldsymbol{k}} \frac{\Lambda_{\boldsymbol{k}}\left(\boldsymbol{q}^{*} ; 0\right)}{\omega_{l}^{2}+\eta_{\boldsymbol{k}}\left(\boldsymbol{q}^{*}, 0\right)^{2}}
\end{aligned}
$$

In this case $\mathcal{L}^{(3)}\left(\boldsymbol{q}^{*} ; i \omega_{l} ; \boldsymbol{q}^{*} ; i \Omega_{m}=0\right)=0$ and thus the diagram (c), (d), (f) and (g) give no contribution. Although we can safely say that $\Delta \chi_{\perp}^{+-}$gives rise to the negative contribution, the sign of the contribution of $\Delta \chi_{/ /}^{+-}$is ambiguous. To settle this problem, it is rather instructive to investigate explicitly the behavior of the integrand of $\mathcal{L}^{(2)}\left(\boldsymbol{q}^{*} ; i \omega_{l} ; \boldsymbol{q}^{*} ; i \Omega_{m}=0\right)$, i.e.

$$
\mathcal{L}_{\boldsymbol{k}}^{(1)}\left(\boldsymbol{q}^{*} ; i \omega_{l} ; \boldsymbol{q}^{*} ; i \Omega_{m}=0\right)=\frac{-\frac{1}{2 T} \cosh ^{-2} \frac{\xi \boldsymbol{k}}{2 T}}{\omega_{l}^{2}+\eta_{\boldsymbol{k}}\left(\boldsymbol{q}^{*} ; 0\right)^{2}}+2 \frac{\eta_{\boldsymbol{k}}\left(\boldsymbol{q}^{*} ; 0\right) \Lambda_{\boldsymbol{k}}\left(\boldsymbol{q}^{*} ; 0\right)}{\left\{\omega_{l}^{2}+\eta_{\boldsymbol{k}}\left(\boldsymbol{q}^{*} ; 0\right)^{2}\right\}^{2}}
$$

As shown in Fig.10, the integrand is positive in almost all the region in the first Brillouin zone. We can see that this behavior sensitively reflects the present Fermi surface given by (12). Thus in the present case both of the diagram (a) and (e) give negative contribution and suppresses the total spin fluctuations, $\chi+\Delta \chi / /+\Delta \chi \perp$, in

the low energy region. We can call this mechanism the low energy dynamical screening effects. From this result, we can see that the coupling of the spin fluctuations on each layer via the enhanced inter-layer exchange scattering processes can dramatically screen the low energy intra-layer spin fluctuations, $\chi(q)$.

\section{Neutron Scattering and NMR in Magnetically Coupled Bi-layer Cuprates}

In the present section we show how the diagonal and off-diagonal spin-spin correlation functions appear in the expression of the neutron scattering cross section and NMR relaxation rates. 


\section{Neutron Scattering Cross Section}

A neutron has magnetic moment $\boldsymbol{\mu}_{N}=-\gamma \mu_{N} \boldsymbol{s}$, where $\boldsymbol{s}$ is the neutron spin. The g-factor $\gamma=1.91$. This magnetic moment at the position $\mathbf{r}_{N}$ induces the magnetic field at the position $\mathbf{r}$ as $\mathbf{B}\left(\mathbf{r}-\mathbf{r}_{N}\right)=\nabla_{\mathbf{r}} \times\left(\boldsymbol{\mu}_{N} \times \frac{\mathbf{r}-\mathbf{r}_{N}}{\left|\mathbf{r}-\mathbf{r}_{N}\right|^{3}}\right)$. Fluctuating electronic spin at the position $\mathbf{r}_{e}$ interacts with this magnetic field. We note that in case of bi-layer compounds, $\mathbf{r}_{e}$ is confined in layer- 1 or layer-2. This interaction can be expressed by $\mathcal{H}_{\text {el-neu }}=-\left(e / m_{e} c\right) \sum_{\mathbf{r}_{e} \in \text { layer-1,2 }} \mathbf{S}\left(\mathbf{r}_{e}, t\right) \cdot \mathbf{B}\left(\mathbf{r}_{e}-\mathbf{r}_{N}\right)$.

The initial and final state of the electron-neutron system can be labeled by | $\boldsymbol{k}_{i} \sigma_{i} E_{i}>$ and $\mid \boldsymbol{k}_{f} \sigma_{f} E_{f}>$ respectively where $\boldsymbol{k} \sigma$ denote a wave number and spin of neutron and $E$ denotes a electronic state. Then the inelestic neutron scattering cross section can be given by 59]

$$
\frac{d \sigma}{d \Omega d \omega}=\left(\frac{m_{N}}{2 \pi \hbar}\right)^{2} \frac{k_{f}}{k_{i}} \sum_{\sigma_{i} \sigma_{f}} \sum_{E_{i} E_{f}} P_{E_{i}} P_{\sigma_{i}}\left|<\boldsymbol{k}_{i} \sigma_{i} E_{i}\right| \mathcal{H}_{e l-n e u}\left|\boldsymbol{k}_{f} \sigma_{f} E_{f}>\right|^{2} \delta \text { (energy), }
$$

where $P_{E_{i}}$ and $P_{\sigma_{i}}$ denote probability factor and the delta function represents energy conservation. By using the fluctuation-dissipation thoerem, we get

$$
\frac{d \sigma}{d \Omega d \omega} \propto \sum_{\alpha \beta}\left(\delta_{\alpha \beta}-\hat{q}_{\alpha} \hat{q}_{\beta}\right) \frac{\Im \chi_{/ /}^{\alpha \beta}(\boldsymbol{q}, \omega)+\Im \chi_{\perp}^{\alpha \beta}(\boldsymbol{q}, \omega)}{1-\exp (-\omega / T)} .
$$

From this result, we can see that both of intra- and inter-layer spin-spin correlation equally contribute to the inelastic neutron scattering cross section.

\section{NMR Longitudinal Relaxation Process}

In the case of YBCO, Hamiltonian for the spin $\mathbf{I}_{m i}$ of the $i$-th ${ }^{63} \mathrm{Cu}(m$ is a layer index) can be written by $\mathcal{H}=-\gamma_{63} \mathbf{H} \cdot\left(\mathbf{1}+{ }^{63} \mathbf{K}^{\text {orb }}\right) \cdot \mathbf{I}_{m i}+\mathcal{H}_{\text {el-nuc }}$, where $\gamma_{63}$ is the g-factor and ${ }^{63} \mathbf{K}^{\text {orb }}$ is the orbital contribution. The interaction between the ${ }^{63} \mathrm{Cu}$ nucler spin and the neighboring electronic spins can be expressed as $\mathcal{H}_{\text {el-nuc }}=$ $\sum_{\mathbf{r}_{j} \in \text { layer-m }} I_{m i}^{\alpha}{ }^{63} A_{i j}^{\alpha \beta} S^{\beta}\left(\mathbf{r}_{j}\right)$. The hyperfine coupling tensor $\mathbf{A}_{i j}$ is proportional to the amplitude of the electronic Bloch function at the nuclear site in question. In case of YBCO, strong hybridization between the neighboring $\mathrm{Cu}$ sites produces the transferhyperfine coupling in the form ${ }^{63} A_{i j}^{\alpha \beta}=\delta_{i j} A^{\alpha \beta}+\delta_{i, j+\hat{a}} B$ 61. The first term indicates the core polarization, while the second term indicates the transfer hyperfine coupling term that is assumed to be isotropic, where $i$ and $j+\hat{a}$ denote the nearest $\mathrm{Cu}$ sites. We note that unless there is the strong hybridyzation between the adjacent layers, we cannot expect the inter-layer spin correlation contributes to the $T_{1}$.

Then the relaxation rate $T_{1}$ can be obtained by [60]

$$
\frac{1}{T_{1}} \propto T \sum_{\boldsymbol{q}}\left|{ }^{63} A_{\boldsymbol{q}}\right|^{2} \frac{\Im \chi_{/ /}^{-+}(\boldsymbol{q}, \omega)}{\omega},
$$

where $\omega$ is the nuclear Larmor frequency. The Fourier transform of the hyperfine coupling for YBCO is given by ${ }^{63} A_{\boldsymbol{q} / /}=A_{/ /}+2 B\left(\cos q_{x} a+\cos q_{y} a\right),{ }^{63} A_{\perp \perp}=A_{\perp}+2 B\left(\cos q_{x} a+\cos q_{y} a\right)$ 
for ${ }^{63} \mathrm{Cu}$ sites. We should stress that only intra-layer spin fluctuations contribute to the NMR longitudinal relaxation. This situation is completely different from the case of the neutron scattering.

\section{Spin Echo Decay Rate}

The spin echo detects the refocused transverse spin components after appropreate pulses. If the nuclear spins flip during the experiment, the spin echo decays. In cuprates, the spin echo decay arises from mutual spin flips caused by the indirect interaction between the nuclear spins via the electronic spin fluctuations. This interaction is the same as the well known RKKY interaction in the case of an electron gas and can be written as $\mathcal{H}_{\text {int }}=-\left(\gamma_{n} \hbar\right)^{2} \sum_{m n}\left\{\sum_{\mathbf{r}_{k} \neq \mathbf{r}_{l} \in \text { layer }-m, n} I_{m i}^{\alpha}{ }^{63} A_{i k}^{\alpha \mu} \Re \chi^{\mu \nu}\left(\mathbf{r}_{k}-\mathbf{r}_{l}\right)^{63} A_{l j}^{\nu \beta} I_{n j}^{\beta}\right\}$, where $\Re \chi^{\alpha \beta}\left(\mathbf{r}_{k}-\mathbf{r}_{l}\right)$ is the static non-local susceptibility. Roughly speaking, the Gaussian decay rate mesures the mean time of nuclear spin flips due to the indirect interaction.

When we approximate the decay of the NMR spin-echo envelop by a Gaussian

[10, 62] as $e^{-\frac{t^{2}}{2 T_{2 G}}}$, the contribution from the intra-layer correlation can be written by

$$
\left(\frac{1}{T_{2 G}}\right)_{/ /}^{2} \propto \sum_{\boldsymbol{q}} A_{\boldsymbol{q} / /}^{2} \Re \chi_{/ / /}^{z z}(\boldsymbol{q}, 0)^{2}-\left\{\sum_{\boldsymbol{q}} A_{\boldsymbol{q} / /} \Re \chi_{/ / /}^{z z}(\boldsymbol{q}, 0)\right\}^{2},
$$

while the contribution from the inter-layer correlation can be written by

$$
\left(\frac{1}{T_{2 G}}\right)_{\perp}^{2} \propto \sum_{\boldsymbol{q}} A_{\boldsymbol{q} / /}^{2} \Re \chi_{\perp}^{z z}(\boldsymbol{q}, 0)^{2} .
$$

We note that in the inter-layer counterpart it is not necessary to eliminate the contribution from the term with $\mathbf{r}_{k}=\mathbf{r}_{l}$, since the adjacend layer are spatially separated. Thus the observed spin echo decay rate can be written in the form

$$
\left(\frac{1}{T_{2 G}}\right)^{2}=\left(\frac{1}{T_{2 G}}\right)_{/ /}^{2}+\left(\frac{1}{T_{2 G}}\right)_{\perp}^{2} .
$$

We note that both of the intra- and inter-layer spin correlation contribute to the spin echo decay rate.

\section{$7 \quad$ Numerical Results and Discussion}

In Fig.11(a) is shown the energy dependence of the retarded counterpart of the commensurate RPA dynamical susceptibility $\chi_{\text {ret }}^{+-}\left(\boldsymbol{q}^{*}, \omega\right)$. The retarded counterpart is obtained by analytic countinuation by using numerical Pade approximation 63]. We fix the chemical potential to $\mu=-0.54$. We note that we do not treat the chemical potential shift due to the interaction in a self consistent manner and as a result the 
carrier density cannot be fixed. We set Hubbard interaction $U=3.25 t$. As temperature becomes lower, the peak around $\omega \sim 0.46 t$ becomes sharp. We can assign this peak to the dynamical nesting arising from the electron-hole excitation energy spectrum $\varepsilon_{\boldsymbol{k}+\boldsymbol{q}^{*}}-\varepsilon_{\boldsymbol{k}}=4 t\left(\cos k_{x} a+\cos k_{y} a\right)$ at the momentum transfer $\boldsymbol{q}^{*}=(\pi / a, \pi / a)$.

In Fig. 11(b), we show the energy dependence of the imaginary part of the low energy total susceptibilities, $\chi_{\text {ret }}^{+-}\left(\boldsymbol{q}^{*}, \omega\right)+\Delta \chi_{/ / r e t}^{+-}\left(\boldsymbol{q}^{*}, \omega\right)+\Delta \chi_{\perp \text { ret }}^{+-}\left(\boldsymbol{q}^{*}, \omega\right)$, that can be detected by neutron scattering experiments. Now $\chi$ denotes the intra-layer RPA spin fluctuation defined by (27). We can see the lower side of the peak is strongly suppressed as the temperature is lowered. We can assign this suppression to dynamical screening due to the developed inter-layer exchange scattering processes.

Concerning the characterisitc energy scale of the apparant-gap, we get $\omega_{g} \sim 0.4 t$ that is too large to quantitatively explain the neutron and NMR experiments. This situation comes from our simple treatment for the intra-layer spin fluctuations. In reality the intra-layer carrier propagator should be renormalized by the strongly developed intra-layer spin flctuations. Then as the temperature is decreased, the loci of the peak in the dynamnical spin excitation spectrum shifts to lower energy side, due to the temperature dependent renormalization factor. So we can say that if we take into account these flctuation effects, the peak moves to the lower energy side and $\omega_{g}$ can also go down to smaller value. To see the mechanism of dynamical screening in detail, in Fig. 12, we show separately the contributions from the diagram (a) and (e) in the case of $T=0.008 t$.

In our scenario, both of the diagonal and off-diagonal spin susceptibility is suppressed due to the dynamical screening effects due to the strongly enhanced interlayer exchange scattering processes, and as a result the intra-layer RPA dynamical susceptibility that is strongly enhanced due to the intra-layer antiferromagnetic spin fluctuations tends to be suppressed. Thus we can get the apparent gap-like structure in the low energy spin excitation spectrum.

Next we consider the NMR longitudinal relaxation rates. As we have shown, in our scenario, the dynamical screening effects leads to the strong suppression of the low energy spin excitations. Then we can expect that NMR relaxation rates should be suppressed. We can see from Fig. 11(b) that actually the slope at $\omega \rightarrow 0$, namely $\lim _{\omega \rightarrow 0} \Im \chi\left(\boldsymbol{q}^{*}, \omega\right) / \omega$, gradually decreases as the temperature decreases. However the suppression of the low energy spin excitation spectrum can be observed even in the simple RPA results in Fig. 11(a). In this case the suppression is due to the dynamical nesting. This situation shows us it is difficult to discuss NMR relaxation only in terms of the commensurate fluctuations.

To clarify this point we performed the momentum integration of the contribution from the diagram (a) with Mila-Rice form factor 61]. The results are shown in Fig. 13. In this case the contribution arising from the fluctuations with $\boldsymbol{q} \neq \boldsymbol{q}^{*}=(\pi / a, \pi / a)$ becomes important since the dynamical nesting effects observed within the intralayer RPA is destroyed at $\boldsymbol{q} \neq \boldsymbol{q}^{*}$. As a result within the intra-layer RPA, $1 / T_{1} T$ monotonically increases as the temperature decreases. By taking into account the bi-layer effects, however, the gap like structures survive since the internal momentum 
of the T-matrices in the diagram (a) is independent of the external momentum and can be enhanced regardress of the deviation of the external momentum from the commensurate momentum. Thus the PSGA behavior can be obtained in the wide region in the momentum space and $1 / T_{1} T$ can detect PSGA in the present scheme.

Finally we comment on the NMR spin echo decay rates in our scheme. As was mentioned in the previous section, spin echo decay rates detect the strength of the indirect interaction. In this case the negative sign coming from the diagram (e) is meaningless, and therefore the development of the contribution of the diagram (e) tends to enhance the spin echo decay rates. As a result, this enhancement cancels the screening effect arising from the diagram (a). Therefore the spin echo decay rate is expected to be enhanced in the low temperature region where the intra-layer spin fluctuations are strongly developed. This situation may be a clue to understand the reason why $1 / T_{1} T$ and $1 / T_{2 G}$ show different temperature dependence in YBCOE.

\section{Concluding Remarks}

In the present paper we proposed a possible mechanism of PSGA in magnetically coupled bi-layer cuprates. We shall summarize a physical picture for the pseudo-spin gap formation in the present scheme. The basic steps are as follows.

STEP 1: In a lightly doped bi-layer cuprate, an intra-layer itinerant electronic system lies just near the antiferromagnetc phase boundary.

STEP 2: The itineracy of the intra-layer system dramatically enhances inter-layer antiferromagnetc coupling. As a result inter-layer coupling becomes temperature dependent and developes dramatically as the temperature decreaces.

STEP 3: Just near the magnetic phase boundary the inter-layer particle-hole ladder becomes very important. Dramatically enhanced inter-layer antiferromagnetic coupling induces inter-layer exchange scattering processes. This process leads to strong attraction for inter-layer spin singlet pair and strong repulsion for inter-layer spin triplet pair. Namely the strong repulsion between parallel spins is strongly enhanced as the intra-layer system goes nearer to the magnetic phase boundary.

STEP 4: Strong repulsion between parallel spins lead to the dynamical screening of the total magnetic excitations in the low energy region. Thus PSGA can be realized in a magnetically coupled bi-layer cuprate.

In the present work, we have not payed our attention to the self consistent renormalization effects. Now we note that the inter-layer exchange scattering processes do not contribute to any vertex correction, since these processes do not af-

\footnotetext{
${ }^{2}$ Now we proceed with numerical calculations to assert this point. We will present the results elsewhere.
} 
fect the number conservation of carriers. Thus it is sufficient to consider the selfenergy effects. When we consider the self consistency, the diagram (a) and (b) in Fig. 9 should be regarded as the self energy diagram. Then the bare green function $\mathcal{G}_{0}(k)$ should be replaced by the dressed one $\mathcal{G}(k)=\left[i \varepsilon_{n}-\xi_{\boldsymbol{k}}-\Sigma(k)\right]^{-1}$, where the self energy is given by $\Sigma(k)=\frac{T}{N} \sum_{Q}\left[\mathcal{T}_{\perp F W}^{\uparrow \uparrow, \uparrow \uparrow}(Q)+\mathcal{T}_{\perp F W}^{\uparrow \perp, \uparrow \downarrow}(Q)\right] \mathcal{G}(k+Q)$. By putting $x(k)=\Re \Sigma(k), z(k)=1-\frac{\Im \Sigma(k)}{\varepsilon_{n}}$, we can write the green function in a form $\mathcal{G}(k)=\frac{1}{z(k)} \frac{1}{i \varepsilon_{n}-\tilde{\xi}_{\boldsymbol{k}}}$, where $\tilde{\xi}_{\boldsymbol{k}}=\frac{\xi_{\boldsymbol{k}}+x(k)}{z(k)}$. Then the irreducible loop in RPA is replaced

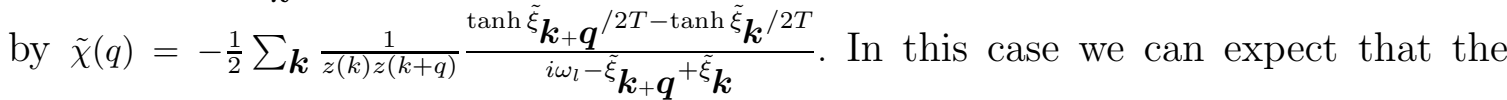
dynamical screening effects in the present context corresponds to the enhancement of mass-renormalization factor $z(k)$ near the antiferromagnetic phase boundary. We may say that in our scenario the mass-renormalization depends sensitevely on $\omega$ and the low energy mass becomes heavier than the high energy mass. If this mechanism really takes place, PSGA is obtained again. We will report the results of sophisticated calculations in the forthcoming paper.

\section{Acknowledgements}

I would like to express my thanks to Professors H.Namaizawa, M.Ogata and H.Fukuyama for valuable discussions and critical comments. I gratefully acknowledge helpful discussion with Dr.Y.Itoh on experimental insights, especially on NMR experiments. Furthermore I would like to thank to Dr.Shiina for useful advice on numerical work.

\section{Appendix: Spin-rotational Symmetry Relation}

Now we show the spin rotational invariance relation in the present scheme by taking into account all the diagrams for the contribution to the longitudinal susceptibility. Necessary diagrams are shown in Fig. 14. Our goal is to show the relation

$$
\begin{aligned}
& \Delta \chi_{/ /}^{+-}(q)=2 \Delta \chi_{/ /}^{z z}(q), \\
& \Delta \chi_{\perp}^{+-}(q)=2 \Delta \chi_{\perp}^{z z}(q),
\end{aligned}
$$

where

$$
\Delta \chi_{m n}^{z z}(q)=\frac{1}{4} \sum_{\sigma}\left[\Delta \chi_{m n}^{\sigma, \sigma}(q)-\Delta \chi_{m n}^{\sigma,-\sigma}(q)\right] .
$$

Contribution to the longitudinal diagonal susceptibility can be obtained through the diagrammatic algebra shown in Fig. 15(a) and we get

$$
\begin{aligned}
& \Delta \chi_{/ /}^{\sigma, \sigma}(q)-\Delta \chi_{/ /}^{\sigma,-\sigma}(q)=\gamma(q)^{2} \frac{T}{N} \sum_{Q}\left\{\mathcal{T}_{\perp F W}^{\uparrow \uparrow, \uparrow}(Q)+\mathcal{T}_{\perp F W}^{\uparrow, \uparrow \uparrow}(Q)\right\} \mathcal{L}^{(1)}(q, Q) \\
& +\gamma(q)^{2} \frac{T}{N} \sum_{Q}\left\{\mathcal{T}_{\perp F W, B W}^{\uparrow \uparrow, \uparrow \uparrow}(Q) \mathcal{T}_{\perp F W, B W}^{\uparrow \uparrow, \uparrow}(Q+q)+\right. \\
& \left.\mathcal{T}_{\perp F W, B W}^{\uparrow \downarrow, \uparrow \downarrow}(Q) \mathcal{T}_{\perp F W, B W}^{\uparrow, \uparrow \downarrow}(Q+q)-\mathcal{T}_{\perp F W, B W}^{\uparrow, \downarrow \uparrow \uparrow}(Q) \mathcal{T}_{\perp F W, B W}^{\uparrow \downarrow, \downarrow \uparrow}(Q+q)\right\}\left\{\mathcal{L}^{(3)}(q, Q)\right\}^{2},
\end{aligned}
$$


where

$\mathcal{T}_{\perp F W, B W}^{\mu \nu, \rho \lambda}(Q) \mathcal{T}_{\perp F W, B W}^{\mu^{\prime} \nu^{\prime}, \rho^{\prime} \lambda^{\prime}}(Q+q) \equiv \mathcal{T}_{\perp F W}^{\mu \nu, \rho \lambda}(Q) \mathcal{T}_{\perp F W}^{\mu^{\prime} \nu^{\prime}, \rho^{\prime} \lambda^{\prime}}(Q+q)+\mathcal{T}_{\perp B W}^{\mu \nu, \rho \lambda}(Q) \mathcal{T}_{\perp B W}^{\mu^{\prime} \nu^{\prime}, \rho^{\prime} \lambda^{\prime}}(Q+q)$

Furthermore the algebra shown in Fig. 15(b) produces the off-diagonal counterpart as

$$
\begin{array}{r}
\Delta \chi_{\perp}^{\sigma \sigma}(q)-\Delta \chi_{\perp}^{\sigma,-\sigma}(q)=-\gamma(q)^{2} \frac{T}{N} \sum_{Q}\left\{\mathcal{T}_{\perp B W}^{\uparrow \uparrow, \uparrow \uparrow}(Q)-\mathcal{T}_{\perp B W}^{\uparrow \downarrow, \uparrow \downarrow}(Q)\right\} \mathcal{L}^{(2)}(q, Q) \\
+\gamma(q)^{2} \frac{T}{N} \sum_{Q}\left\{\mathcal{T}_{\perp F W, B W}^{\uparrow \uparrow, \uparrow}(Q) \mathcal{T}_{\perp F W, B W}^{\uparrow \uparrow, \uparrow \uparrow}(Q+q)-\right. \\
\left.\mathcal{T}_{\perp F W, B W}^{\uparrow, \uparrow \downarrow}(Q) \mathcal{T}_{\perp B W, F W}^{\uparrow \downarrow, \uparrow \downarrow}(Q+q)-\mathcal{T}_{\perp F W, B W}^{\uparrow \downarrow, \downarrow \uparrow}(Q) \mathcal{T}_{\perp B W, F W}^{\uparrow \downarrow, L \uparrow}(Q+q)\right\} \mathcal{L}^{(3)}(q, Q) \mathcal{L}^{(3)}(-q,-Q),(89
\end{array}
$$

where

$\mathcal{T}_{\perp F W, B W}^{\mu \nu, \rho \lambda}(Q) \mathcal{T}_{\perp B W, F W}^{\mu^{\prime} \nu^{\prime}, \rho^{\prime} \lambda^{\prime}}(Q+q) \equiv \mathcal{T}_{\perp F W}^{\mu \nu, \rho \lambda}(Q) \mathcal{T}_{\perp B W}^{\mu^{\prime} \nu^{\prime}, \rho^{\prime} \lambda^{\prime}}(Q+q)+\mathcal{T}_{\perp B W}^{\mu \nu, \rho \lambda}(Q) \mathcal{T}_{\perp F W}^{\mu^{\prime} \nu^{\prime}, \rho^{\prime} \lambda^{\prime}}(Q+q)$

From now on we omitt the indices $F W$ and $B W$, since these indices are not essential for the spin rotational symmetry. By noting the spin rotaional invariance relation for the exchange scattering T-matrixes, (60),

$$
\mathcal{T}_{\perp}^{\uparrow \uparrow, \uparrow \uparrow}(Q)-\mathcal{T}_{\perp}^{\uparrow \downarrow, \downarrow \uparrow}(Q)=\mathcal{T}_{\perp}^{\uparrow \downarrow, \uparrow \downarrow}(Q)
$$

we can rewrite the terms in the braces in (88) as,

$$
\begin{array}{r}
\mathcal{T}_{\perp}^{\uparrow \uparrow, \uparrow \uparrow}(Q) \mathcal{T}_{\perp}^{\uparrow \uparrow, \uparrow \uparrow}(Q+q)+\mathcal{T}_{\perp}^{\uparrow \downarrow, \uparrow \downarrow}(Q) \mathcal{T}_{\perp}^{\uparrow \downarrow, \uparrow \downarrow}(Q+q)-\mathcal{T}_{\perp}^{\uparrow \downarrow, \downarrow \uparrow}(Q) \mathcal{T}_{\perp}^{\uparrow \downarrow, \downarrow \uparrow}(Q+q) \\
=\mathcal{T}_{\perp}^{\uparrow \uparrow, \uparrow \uparrow}(Q) \mathcal{T}_{\perp}^{\uparrow \uparrow, \uparrow \uparrow}(Q+q)+\mathcal{T}_{\perp}^{\uparrow \downarrow, \uparrow \downarrow}(Q) \mathcal{T}_{\perp}^{\uparrow \downarrow, \uparrow \downarrow}(Q+q) \\
-\left\{\mathcal{T}_{\perp}^{\uparrow \uparrow, \uparrow \uparrow}(Q)-\mathcal{T}_{\perp}^{\uparrow \downarrow, \uparrow \downarrow}(Q)\right\}\left\{\mathcal{T}_{\perp}^{\uparrow \uparrow, \uparrow \uparrow}(Q+q)-\mathcal{T}_{\perp}^{\uparrow \downarrow, \uparrow \downarrow}(Q+q)\right\} \\
=2\left\{\mathcal{T}_{\perp}^{\uparrow, \uparrow \uparrow}(Q) \mathcal{T}_{\perp}^{\uparrow \downarrow, \uparrow \downarrow}(Q+q)+\mathcal{T}_{\perp}^{\uparrow \downarrow, \uparrow \downarrow}(Q) \mathcal{T}_{\perp}^{\uparrow \uparrow, \uparrow \uparrow}(Q+q)\right\}
\end{array}
$$

and similarly in (89),

$$
\begin{array}{r}
\mathcal{T}_{\perp}^{\uparrow \uparrow, \uparrow \uparrow}(Q) \mathcal{T}_{\perp}^{\uparrow \uparrow, \uparrow \uparrow}(Q+q)-\mathcal{T}_{\perp}^{\uparrow \downarrow, \uparrow \downarrow}(Q) \mathcal{T}_{\perp}^{\uparrow \downarrow, \uparrow \downarrow}(Q+q)-\mathcal{T}_{\perp}^{\uparrow \downarrow, \downarrow \uparrow}(Q) \mathcal{T}_{\perp}^{\uparrow \downarrow, \downarrow \uparrow}(Q+q) \\
=\left\{\mathcal{T}_{\perp}^{\uparrow \downarrow, \downarrow \uparrow}(Q+q)+\mathcal{T}_{\perp}^{\uparrow \downarrow, \uparrow \downarrow}(Q+q)\right\}\left\{\mathcal{T}_{\perp}^{\uparrow \downarrow, \downarrow \uparrow}(Q)+\mathcal{T}_{\perp}^{\uparrow \downarrow, \uparrow \downarrow}(Q)\right\} \\
\quad-\mathcal{T}_{\perp}^{\uparrow \downarrow, \uparrow \downarrow}(Q) \mathcal{T}_{\perp}^{\uparrow \downarrow, \uparrow \downarrow}(Q+q)-\mathcal{T}_{\perp}^{\uparrow \downarrow, \downarrow \uparrow}(Q) \mathcal{T}_{\perp}^{\uparrow \downarrow, \downarrow \uparrow}(Q+q) \\
=2\left\{\mathcal{T}_{\perp}^{\uparrow \downarrow, \downarrow \uparrow}(Q) \mathcal{T}_{\perp}^{\uparrow \downarrow, \uparrow \downarrow}(Q+q)+\mathcal{T}_{\perp}^{\uparrow \downarrow, \uparrow \downarrow}(Q) \mathcal{T}_{\perp}^{\uparrow \downarrow, \downarrow \uparrow}(Q+q)\right\}
\end{array}
$$

Thus we get the results

$$
\begin{array}{r}
\Delta \chi_{/ /}^{\sigma, \sigma}(q)-\Delta \chi_{/ /}^{\sigma,-\sigma}(q)= \\
\gamma(q)^{2} \frac{T}{N} \sum_{Q}\left\{\mathcal{T}_{\perp F W}^{\uparrow \uparrow, \uparrow \uparrow}(Q)+\mathcal{T}_{\perp F W}^{\uparrow \downarrow, \uparrow \downarrow}(Q)\right\} \mathcal{L}^{(1)}(q, Q) \\
2 \gamma(q)^{2} \frac{T}{N} \sum_{Q}\left\{\mathcal{T}_{\perp}^{\uparrow \uparrow, \uparrow \uparrow}(Q) \mathcal{T}_{\perp}^{\uparrow \downarrow, \uparrow \downarrow}(Q+q)+\mathcal{T}_{\perp}^{\uparrow \downarrow, \uparrow \downarrow}(Q) \mathcal{T}_{\perp}^{\uparrow \uparrow, \uparrow \uparrow}(Q+q)\right\}\left\{\mathcal{L}^{(3)}(q, Q)\right\}^{2},
\end{array}
$$


and

$$
\begin{array}{r}
\Delta \chi_{\perp}^{\sigma \sigma}(q)-\Delta \chi_{\perp}^{\sigma,-\sigma}(q)=-2 \gamma(q)^{2} \frac{T}{N} \sum_{Q} \mathcal{T}_{\perp}^{\uparrow \downarrow, \downarrow \uparrow}(Q) \mathcal{L}^{(1)}(q, Q) \\
+2 \gamma(q)^{2} \frac{T}{N} \sum_{Q}\left\{\mathcal{T}_{\perp}^{\uparrow \downarrow, \downarrow \uparrow}(Q) \mathcal{T}_{\perp}^{\uparrow \downarrow, \uparrow \downarrow}(Q+q)\right. \\
\left.+\mathcal{T}_{\perp}^{\uparrow \downarrow, \uparrow \downarrow}(Q) \mathcal{T}_{\perp}^{\uparrow \downarrow, \downarrow \uparrow}(Q+q)\right\} \mathcal{L}^{(3)}(q, Q) \mathcal{L}^{(3)}(-q,-Q),
\end{array}
$$

These expressions are exactly twice the corresponding transverse susceptibility, 69) and (69). Thus we obtain the spin rotational symmetry relation (87). 


\section{References}

[1] P.W.Anderson: Science235(1987)1196.

[2] A.P.Kampf: Phys.Rep.249(1994)219.

[3] H.Yasuoka, T.Imai and T.Shimizu:Strong Correlation and Superconductivity,ed. H.Fukuyama,S.Maekawa,A.P.Malozemoff (Springer,Berlin,1989)Vol.89,p.254.

[4] M.Takigawa: Phys.Rev.B49(1994)4158.

[5] M.Takigawa, A.P.Reyes, P.C.Hammel, J.D.Tompson, R.H.Heffner,Z.Fisk and K.C.Ott: Phys.Rev.B43(1991)247.

[6] H.Alloul, T.Ohno, and P.Mendels: Phys.Rev.Lett63(1989)1700.

[7] W.W.Warren, R.E.Walstedt, G.F.Brennertd, R.J.Cava, R.Tycho, R.E.Bell and G.Dabbagh: Phys.Rev.Lett62(1989)1193.

[8] T.Imai,H.Yasuoka,T.Shimizu, Y.Ueda, K.Yoshimura and K.Kosuge: Physica C162164(1989)169.

[9] T.Imai, C.P.Slichter, A.P.Paulikas and B.Veal: Phys.Rev.B47(1993)9158.

[10] Y.Itoh, H.Yasuoka, Y.Fugiwara, Y.Ueda, T.Machi, I.Tomeno, K.Tai, N.Koshizuka and S.Tanaka: J.Phys.Soc.Jpn.61(1992)1287.

[11] M.Matsumura, Y.Sakamoto, T.Fushihara, Y.Itoh,and H.Yamagata: J.Phys.Soc.Jpn.64(1995)721.

[12] H.Zimmermann, M.Mali, I.Mangelschots, J.Roos, L.Paul, D.Brinkmann, J.Karpinski, S.Rusiecki and E.Kaldis: J.Less-Common Metals 154-165(1990)138.

[13] T.Machi,I.Tomeno,T.Miyatake,N.Koshizuka,S.Tanaka,T.Imai and H.Yasuoka: PhysicaC173(1991)32.

[14] S.Kambe, T.Machi, I.Tomeno, H.Yasuoka, A.Hayashi, U.Ueda: J.Phys.Soc.Jpn.63(1994)3481.

[15] R.Stern, M.Mali, I.Mangelschots, J.Roos, D.Brinkmann, J-Y.Genoud, T.Graf and J.Muller: Phys.Rev.B50(1994) 426.

[16] A.Goto, H.Yasuoka, K.Otzschi, and Y.Ueda: J.Phys.Soc.Jpn.64(1995)367.

[17] R.E.Walstedt, R.F.Bell and D.B.Mitzi: Phys.Rev.B44(1991)7760.

[18] W.Schnelleet al.: PhysicaC161(1991)1287.

[19] J.Rossat-Mignod, L.P.Regnault, P.Bourges, P.Burlet, C.Vettier, J.Y.Henry: PhysicaB192(1993)109.

[20] J.X.Boucherle, J.Y.Henry, R.J.Papoular, J.Rossat-Mignod, J.Schweizer, F.Tasset, G.Uimin: PhysicaB192(1993)25.

[21] J.Rossat-Mignod ,L.P.Regnault, P.Bourges, C.Vettier, P.Burlet: PhysicaB186-188(1993)1.

[22] B.J.Sternlieb, G.Shirane, J.M.Tranquada, M.Sato, and S.Shamoto: Phys.Rev.B47(1993)5320.

[23] H.Yasuoka: Solid State Physics Vol.30(1995)859.

[24] K.Yamada, S.Wakimoto, G.Shirane, C.H.Lee, M.A.Kastner, S.Hosoya, M.Greven, Y.Endoh,and R.J.Birgeneau: preprint

[25] S.Kambe, H.Yasuoka, A.Hayashi and Y.Ueda: Phys.Rev.B47(1993)2825.

[26] Y.Itoh, H.Yasuoka, A.Hayashi and Y.Ueda: J.Phys.Soc.Jpn.6322(1994).

[27] A.J.Millis, and H.Monien: Phys.Rev.Lett.70(1993)2810-13.

[28] N.F.Berk, and J.R.Schrieffer: Phys. Rev. Lett.17(1966)433;S.Doniach, and S.Engelsberg: Phys. Rev. Lett.17(1966)750;P.W.Anderson, and W.F.Brinkmann: Phys. Rev. Lett.30(1973)1108;S.Nakajima, Prog. Theor. Phys.50(1973)1101.

[29] N.Bulut, and D.J.Scalapino: Phys.Rev.Lett.68(1992)706.

[30] N.Bulut, and D.J.Scalapino: Phys. Rev. B47(1993)3419.

[31] S.Wermbter and L.Tewordt: Phys. Rev.B.43(1991)10530.

[32] T.Moriya and A.Kawabata: J.Phys.Soc.Jpn.34(1973)639, T.Moriya and A.Kawabata: J.Phys.Soc.Jpn.35(1973)669

[33] A.Kawabata: J.Phys. F4(1974)1477 
[34] T.Moriya, and K.Ueda: J.Phys.Soc.Jpn.63(1994)1871.

[35] N.E.Bickers and D.J.Scalapino: Annals of Phys.193(1989)206.

[36] J.W.Serene and D.W.Hess: Phys. Rev.B.44(1991)3391.

[37] A.J.Millis, H.Monien and D.Pines: Phys. Rev.B.42(1990)167.

[38] F.C.Zhang and T.M. Rice: Phys. Rev.B.37 (1988)3754.

[39] T.Tanamoto, H.Kohno, H.Fukuyama: J.Phys.Soc.Jpn.60(1991)3072;Tanamoto.T, Kohno.H, Fukuyama.H:J.Phys.Soc.Jpn.62(1993)717; Tanamoto.T, Kohno.H, Fukuyama.H: J.Phys.Soc.Jpn.63(1994)2739.

[40] M.J.Lercher, and J.M.Wheatley: Phys. Rev.B49(1994)736.

[41] B.Normand, H.Kohno, and H.Fukuyama: J.Phys.Soc.Jpn.64(1995)3903.

[42] L.B.Ioffe and A.I.Larkin: Phys. Rev.B.39(1989)8988.

[43] P.A.Lee and N.Nagaosa: Phys. Rev.B.46(1992)5621.

[44] Ubbens.M.U, Lee.P.A: Phys. Rev.B49(1994)6853.

[45] B.L Altshuler and L.B Ioffe: Solid State Commun.82(1992)253.

[46] Ubbens.M.U, and Lee.P.A: Phys. Rev.B50(1994)438.

[47] X-G.Wen, and P.A.Lee: Phys. Rev. Lett.76(1996)503.

[48] L.B.Ioffe, and A.I.Larkin, A.J.Millis, and B.L.Altshuler: Pis'ma Zh. Eksp. Teor. Fiz.59(1994)65.

[49] J.Yu and A.J.Freeman: J.Chem.Solids 52(1991)1351

[50] J.M.Tranquada, G.Shirane, B.Keimer, Shamoto.S, M.Sato.M: Phys. Rev.B40(1989)4503

[51] S.Shamoto, M.Sato.M, J.M.Tranquada, B.Sternlieb, and G.Shirane: Phys.Rev.B48(1993)13817

[52] M.Grüninger, J.Münzel, A.Gaymann, A.Zibol, and H.P.Geserich: preprint.

[53] P.A.Wolff: Phys. Rev.120(1960)814.

[54] J.Kishine: Prog.Theoret.Phys64(1995)543.

[55] L.G.Aslamazov and A.I.Larkin: Soviet Phys.Solid State, 10(1968)875.preprint

[56] K.Maki: Progr.Theoret.Phys.39(1968)897.

[57] B.R.Patton and L.J.Sham: Phys. Rev. Lett.31(1973)631; B.R.Patton and L.J.Sham: Phys. Rev. Lett.33(1974)638.

[58] S-K.Ma, M.T.Beal-Monod, and D.R.Fredkin: Phys.Rev.174(1968)227.

[59] W.Marshall, R.D.Lowde: Rep.Prog.Phys.31(1968)705

[60] T.Moriya: J.Phys.Soc.Jpn.18(1963)516.

[61] F.Mila and T.M.Rice: Phys. Rev.B.40(1989)11382.

[62] C.Pennington and C.P.Slichter: Phys. Rev. Lett.61(1992)1287;D.Thelen and D.Pines: Phys. Rev.B.49(1994)3528.

[63] R.W.Haymaker, and L.Schlessinger in The Pade Approximation in Theoretical Physics Chap.11(Academic Press, 1970) 


\section{Figure Captions}

Fig. 1: Magnetic unit cell of $\mathrm{YBa}_{2} \mathrm{Cu}_{3} \mathrm{O}_{6}$. Filled and open circles denote antiparalle spins at planar $\mathrm{Cu}^{2+}$ sites, while shaded circles denote nonmagnetic $\mathrm{Cu}^{1+}$ ions.

Fig. 2: Fundamental processes induced by the inter-layer magnetic interaction;

(a) The scattering process with spin flip (type-A),

(b) the scattering processes between parallel spins (type-B) and

(c) the scattering processes between anti-parallel spins (type-C).

The straight and wavy line represent respectively the green's function of an itinerant carrier and the inter-layer antiferromagnetic interaction. Here the thick and thin line correspond to an carrier in the layer-1 and the layer-2 respectively.

Fig. 3: Enhancement of the inter-layer exchange interaction vertices due to the intralayer RPA spin fluctuations.

(a): The intra-layer transverse spin fluctuations enhances the Type-A scattering vertex.

(b), (c): The intra-layer longitudinal spin fluctuations enhances both of the Type-B and Type-C scattering vertex. The straight and wavy line represent respectively the green's function of an itinerant carrier and the inter-layer antiferromagnetic interaction. The dotted line represents the intra-layer Hubbard interaction.

Fig. 4: These figures show how the intra-layer spin fluctuations in different layer couple to each other via the inter-layer Heisenberg interaction. The intra-layer transverse spin fluctuations can couple to each other via the type-a inter-layer scattering channel, as is shown in (a). On the other hands the intra-layer longitudinal spin fluctuations can couple to each other via both of the type-b and type-c inter-layer scattering channel, as is shown in (b) and (c). The shaded triangle in the right hand side represents the vertex enhancement due to the intra-layer spin fluctuations where $\gamma(q)$ is the Stoner factor. We can see that the inter-layer interaction is enhanced through the double Stoner factor and strongly enhanced.

Fig. 5: The $n$-th order scattering processes for three scattering channels corresponding to the different spin dependent scattering channel.

(a) the T-matrix that represents the electron-hole exchange scattering process between untiparallel spin,

(b) the T-matrix that represents the electron-hole exchange scattering process between parallel spin, and

(c) the auxiliary T-matrix that consists of only B-type scattering channel.

Fig. 6: Restrictions coming from the momentum tansfer. Since the triangle vertex is enhanced around the momentum transfer $\mathbf{q} \sim \mathbf{q}^{*}$, the incoming momentum( $\left.\mathbf{q}\right)$ and outgoing momentum $\left(\mathbf{k}^{\prime}\right)$ of an itinerant carrier are restricted.

Case (a): When $\mathbf{k}-\mathbf{k}^{\prime} \sim \mathbf{q}^{*}$ (backward scattering) $\Delta$, only the processes including odd number of triangles are enhanced.

Case (b): When $\mathbf{k}-\mathbf{k}^{\prime \prime} \sim 0$ (forward scattering), only the processes including even number of triangles are enhanced. 
Fig. 7: Behavior of T-matrices in the singlet and triplet channel as a function of $\Delta \frac{3}{4} \mathcal{J}_{\perp}(T) \chi_{0}$. Each line represents the forward processes and backward processes distinctively.

Fig. 8: The temperature dependence of the averaged inter-layer interaction $\mathcal{J}_{\perp}(T)$ for $U=3.25 t$ and $J_{0 \perp}=0.08 t$ for the chemical potential $\mu=-0.54$.

Fig.9: The contributions to the transverse susceptibility arising from the enhanced inter-layer exchange scattering process. The graph (a), (b), (c), (d) contribute to the diagonal susceptibility and the graph (e), (f), (g) contribute to the off-diagonal susceptibility. The straight and broken lines represent the propagator of the carrier in different layers.

Fig. 10: The behavior of the integrand in the coupling function $\mathcal{L}_{k}^{(1)}\left(\boldsymbol{q}^{*} ; i \omega_{l} ; \boldsymbol{q}^{*} ; i \Omega_{m}=\right.$ 0 ) in the first quadrant of the Brillouin zone at temperature $T=0.06 t$ and $T=0.01 t$. We set $l=1$ and $\mu=-0.54 t$.

Fig. 11: (a) Imaginary parts of the intra-layer RPA susceptibility for various temperatures $T$.

(b)The retarded counterpart of the total susceptibilities $\chi_{\text {ret }}^{+-}\left(\boldsymbol{q}^{*} ; \omega\right)$ for various temperatures $T$. We fixed the chemical potential to $\mu=-0.54$. We set $U=3.25 t$ and $J_{0 \perp}=0.08 t$.

Fig. 12: The retarded counterpart of the dynamical susceptibility. We show contributions from diagram (a) and (e) separately. [RPA+Diagram(a)] gives the diagonal susceptibility that contribute to NMR longitudinalrelaxation. [RPA+Diagram(a), (e)] is the sum of diagonal and off-diagonal susceptibility that contribute to the neutron scattering experiments.

Fig. 13: NMR longitudinal relaxation rate. The dashed line represents the result from intra-layer RPA, while the solid line represents the result when the inter-layer effects are taken into account.

Fig. 14: Diagrams that are necessary to guarantee the spin rotaional invariance relation when we include the coupling arising from enhanced inter-layer exchange scattering processes;

(a) for the diagonal process, and

(b) for the off-diagonal process. 